Published in final edited form as:

Savateev, A., Pronkin, S., Willinger, M. G., Antonietti, M., \& Dontsova, D. (2017). Towards Organic Zeolites and Inclusion Catalysts: Heptazine Imide Salts Can Exchange Metal Cations in the Solid State. Chemistry - An Asian Journal, 12(13), 1517-1522.

doi:10.1002/asia.201700209.

\title{
Towards organic zeolites and inclusion catalysts: heptazine imide salts can exchange metal cations in the solid state
}

Savateev, A., Pronkin, S., Willinger, M. G., Antonietti, M., \& Dontsova, D.

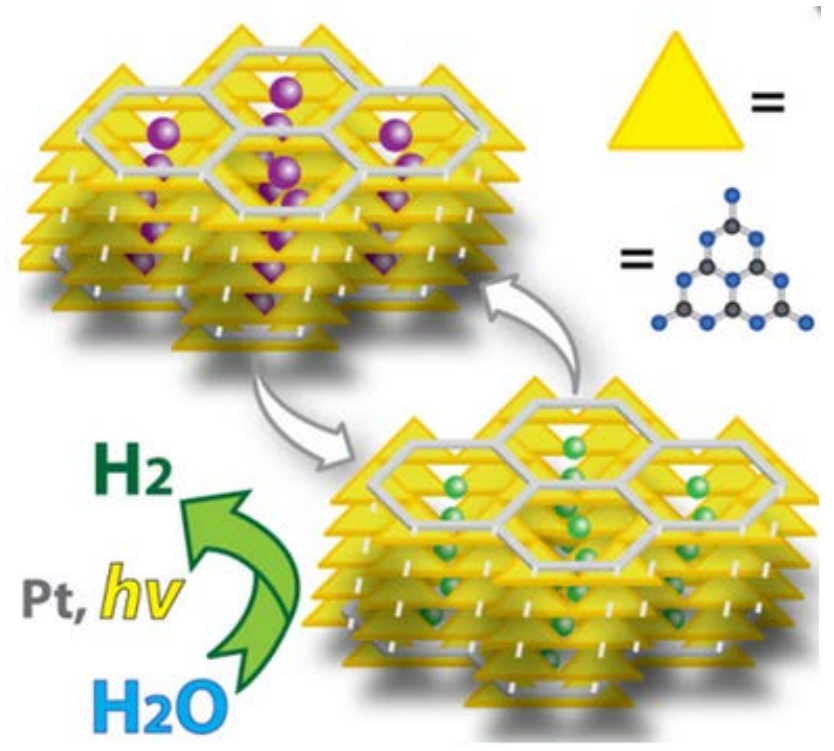

Worth its salt: A variety of heptazine imide salts and a free base can be easily prepared by a simple ion exchange starting from the synthesized potassium derivative. Thus, various mono- and bivalent cations can be incorporated in the structure that resembles the behavior of zeolites. The photocatalytic activity in visible-light-driven hydrogen evolution is shown to depend on the nature of the introduced cation and reaches its maximum in the case of the Mg-salt.

This article may be used for non-commercial purposes in accordance with Wiley Terms and Conditions for Self-Archiving. 


\title{
Towards organic zeolites and inclusion catalysts: heptazine imide salts can exchange metal cations in the solid state
}

\author{
Aleksandr Savateev ${ }^{1}$, Sergey Pronkin ${ }^{2}$, Marc Willinger ${ }^{1,3}$, Markus Antonietti ${ }^{1}$, and Dariya \\ Dontsova $^{1^{*}}$
}

\begin{abstract}
Highly crystalline potassium (heptazine imides) were prepared by the thermal condensation of substituted 1,2,4triazoles in eutectic salt melts. These semiconducting salts are already known to be highly active photocatalysts, e.g. for the visible light driven generation of hydrogen from water. Herein, we show that within the solid state structure, potassium ions can be exchanged to other metal ions while the crystal habitus is essentially preserved.
\end{abstract}

\section{Introduction}

Insertion of ions into solid state structures or exchange of counterions in porous crystalline frameworks is traditionally a domain of inorganic chemistry. For instance, intercalation of Li-ions into $\mathrm{CoO}_{2}$ under reduction of the inorganic frame is the operation principle of the cathode of the lithium battery [1, 2] and exchange of counterions within the crystalline frameworks of charged zeolites allows fine tuning of catalytic activity and size selectivity. Cases of ion insertion or ion exchange in preformed covalent, "organic" crystals are however rare and mostly based on extended polymer frameworks [3].

Recently, carbon nitride materials with a $\mathrm{C} / \mathrm{N}$ stoichiometry close to $\mathrm{C}_{3} \mathrm{~N}_{4}$ such as graphitic carbon nitride g-CN [4, 5] or the more regular poly(triazine imide) $[6,7]$ have emerged as efficient photocatalysts for water reduction and other photocatalytic applications. [8-10] Especially for poly(triazine imide) it is already known that diverse anions could be intercalated [11], while however the diverse ionic species had to be synthesized in different preparations. In addition, intercalation of chloride and bromide changes the gallery heights of the graphitic stacking of the crystals from $0.338 \mathrm{~nm}$ to $0.352 \mathrm{~nm}$, a remarkably large change for a covalent crystal. The intercalated ions were also not accessible from the outside, i.e. base treatment did not result in neutralization or exchange of the halogen ions, except when destroying the crystals.

In order to overcome problems with missing stability and missing crystallinity, we have recently prepared another member of the broader carbon nitride family, poly (heptazine imide) (PHI). [12] $\mathrm{PHI}$ has the advantage of a significantly improved crystallinity, improved electronic conductivity due to the presence of negatively charged nitrogen atoms, and in the context of the present discussion, much larger pores to host in this case metal cations as the counterions. In a wider sense and due to the presence of negatively charged nitrogen atoms in a carbon framework coordinated to a metal cation, poly(heptazine imides) can be considered as wider analogues of porphyrin metal complexes (Scheme 1), whose physicochemical properties are greatly influenced by the central metal cation (for example, light absorption of heme and chlorophyll). First TEM analyses also suggest that the ions are staggered in highly organized channels, i.e, the crystals pack by separating covalent and ionic entities in separated microphases. This is well known from the so-called ionic self-assembly. [13]
1 Max-Planck Institute of Colloids and Interfaces, Department of Colloid Chemistry, Research Campus Golm, 14424 Potsdam, Germany.

${ }^{2}$ Institut de Chimie et des Procédés pour l'Energie, l'Environnement et la Santé (ICPEES), ECPM, CNRS-Université de Strasbourg (UdS) UMR 7515, 25, rue Becquerel, 67087 Strasbourg, France.

${ }^{3}$ Fritz Haber Institute of the Max Planck Society, Department of Inorganic Chemistry, 14195 Berlin, Germany 


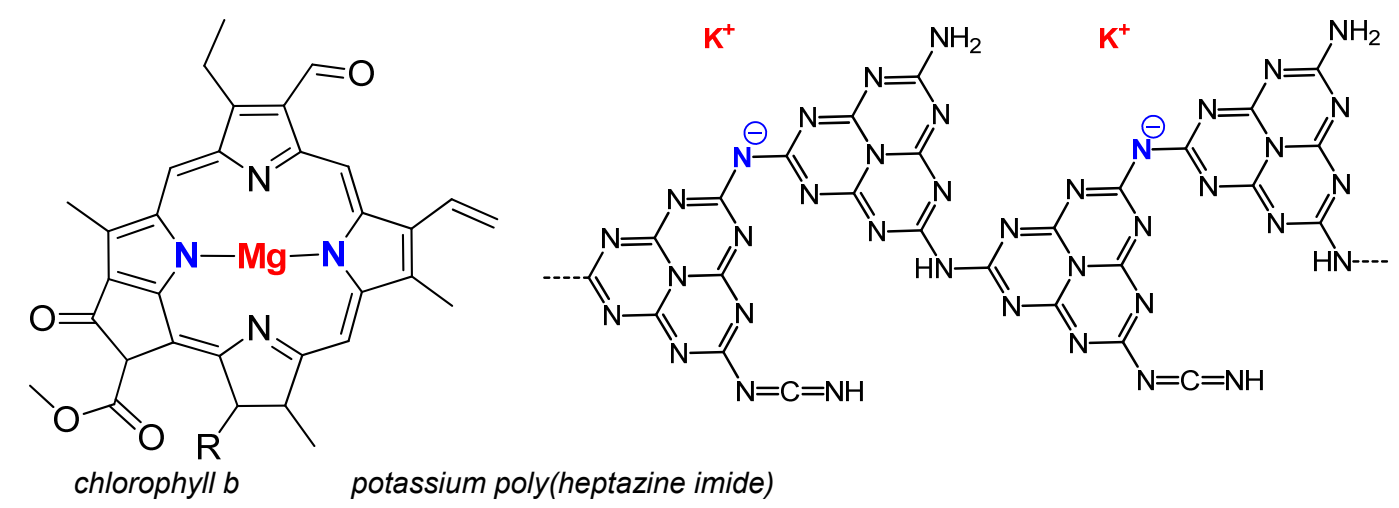

Scheme 1. Chemical structures of chlorophyll $b$ and a schematic potassium poly(heptazine imide). Note that the degree of polymerization, edge termination sites as well as local connectivity motifs are idealized, while equilibrium charge density suggests one potassium counterion per two heptazine units.

So far, only $\mathrm{PHI}$ with $\mathrm{K}^{+}$as counter ions was synthesized, which is essentially due to the choice of the eutectic salt melt, $\mathrm{LiCl} / \mathrm{KCl}$, as a reaction medium. We performed aberration corrected high resolution electron microscopy on these samples and found an unexpected local structure where heptazine units and salt units are found always stacked on top of each other, i.e. ionic

a)

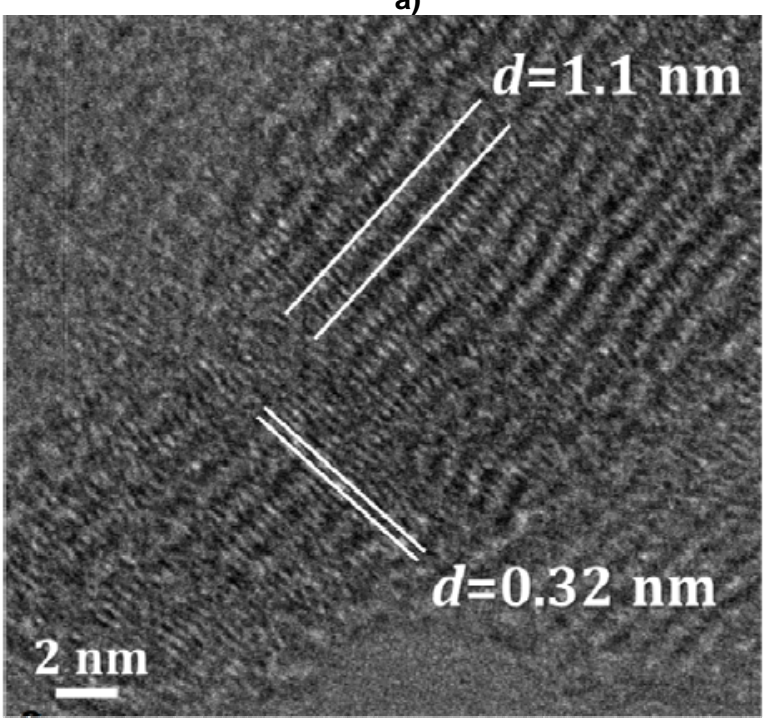

species and covalent heptazine species microphaseseparate in different stacks and channels. This is illustrated in Figure 1. It is clearly seen that the aromatic units stack with a very tight packing with $0.31-0.32 \mathrm{~nm}$ distance, while in the perpendicular direction electron rich (black) ion channels and the heptazine stacks alternate with about 1 $\mathrm{nm}$ periodicity. b)

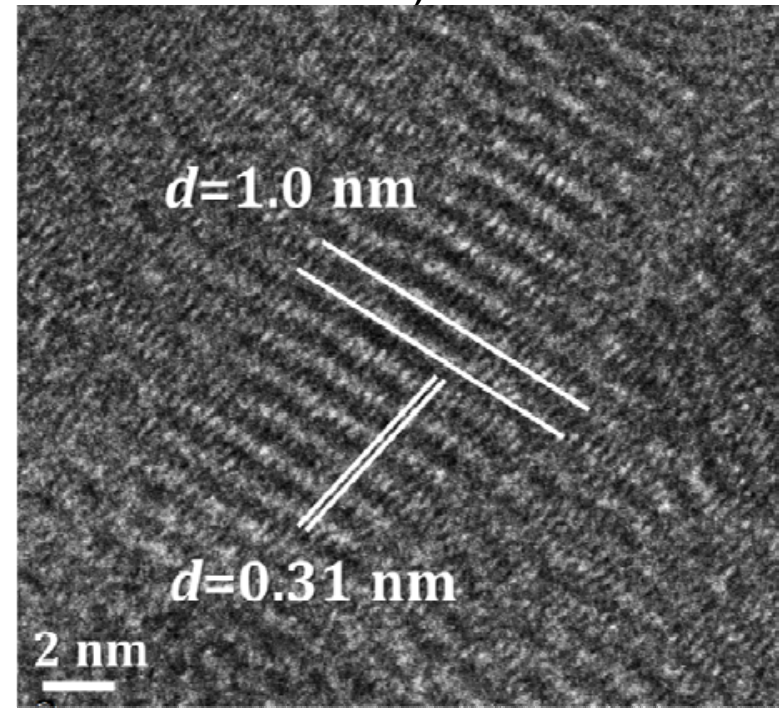

Figure 1. HR-TEM images of PHI-K illustrating the similarity of the material with zeolitic channel compounds, while following the graphic packing of sheetlike organics.

Based on this structure, we show in this contribution that those $\mathrm{PHI}$ salt crystals are able to exchange the channel based metal cations easily while essentially keeping the solid state packing of the organic aromatic structure. Due to this possibility, $\mathrm{PHI}-\mathrm{K}$ can be regarded as a porous material active in solution and as a solid state ion conductor, which, in turn, offers possibilities for post-synthetic modifications. We selected the metal cation exchange in $\mathrm{PHI}-\mathrm{K}$ in order to tune the physicochemical properties of this new type of semiconductor. A series of $\mathrm{PHI}$ related materials containing alkaline ( $\mathrm{Li}, \mathrm{Na}, \mathrm{Cs})$, earth-alkaline (Mg, $\mathrm{Ca})$, and transition metal $(\mathrm{Ni}, \mathrm{Co}, \mathrm{Ag}, \mathrm{Zn})$ cations was prepared. The properties of the resulting PHI-M materials, in particular their catalytic reactivity and conductivity, can be easily varied by introduction of the desired metal cation. 


\section{Results and discussion}

Acid-base behaviour and structural preservation through protonation. Unlike small organic imides such as potassium hexamethyldisilazanide, lithium di-isopropyl amide, lithium tetramethylpiperidide, etc., which are sensitive to both air and moisture, $\mathrm{PHI}$ as a weak acid is an imide completely stable in aqueous media. No significant bond dissolution occurs either in neutral or in basic $(0.1 \mathrm{M}$ $\mathrm{NaOH}, \mathrm{pH}=13$ ) media. This stability of $\mathrm{PHI}$ is due to the resonance stabilization and the electron donating character of the heptazine rings (Scheme S1) and is indeed similar to the well-known trifluoromethylsulfonimide (TFSI) or the porphyrin system.
With $\mathrm{PHI}$ being a weak acid, $\mathrm{K}^{+}$ions can however be easily exchanged by protons at $\mathrm{pH}$ values lower than the $\mathrm{pKa}$ using diluted $(0.1$ or $1.0 \mathrm{M})$ hydrochloric acid solutions. This can be supported by EDX analysis of the protonated acid, denoted as PHI-A (Table S1). The removal of $\mathrm{K}^{+}$ions from the crystals is fully reversible, and treatment of PHI-A with $2.0 \mathrm{M} \mathrm{KOH}$ solution yields a sample $\mathrm{PHI}-\mathrm{B}$, which is in principle similar to the original PHI-K. Our earlier XPS studies have shown that the replacement of $\mathrm{K}^{+}$ions by protons does not lead to the loss of the negative charges at nitrogen atoms [14] suggesting that the protons are not bound to specific sites in the $\mathrm{PHI}$ crystals, i.e. the structure stays Coulombic. This already points to very interesting, zeolite-like activities of the free acid form in catalysis and the presence of proton conductive channels. a)

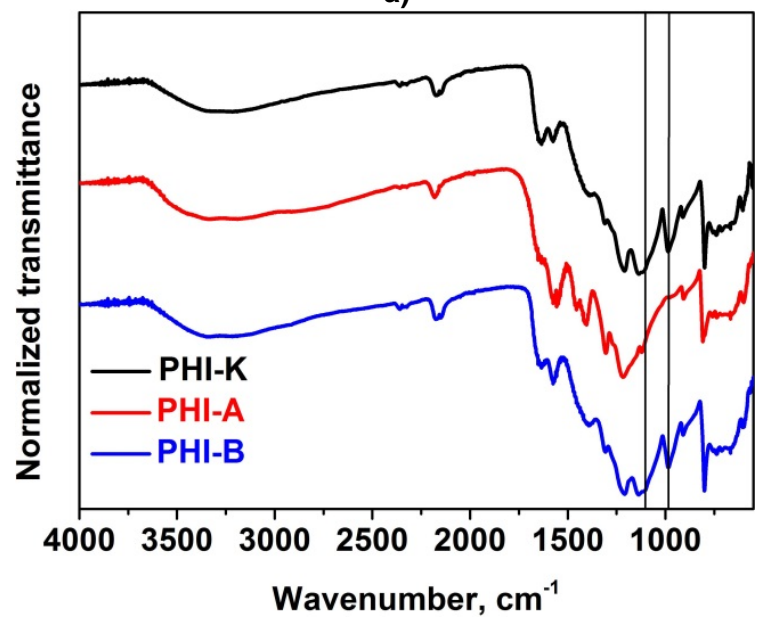

b)

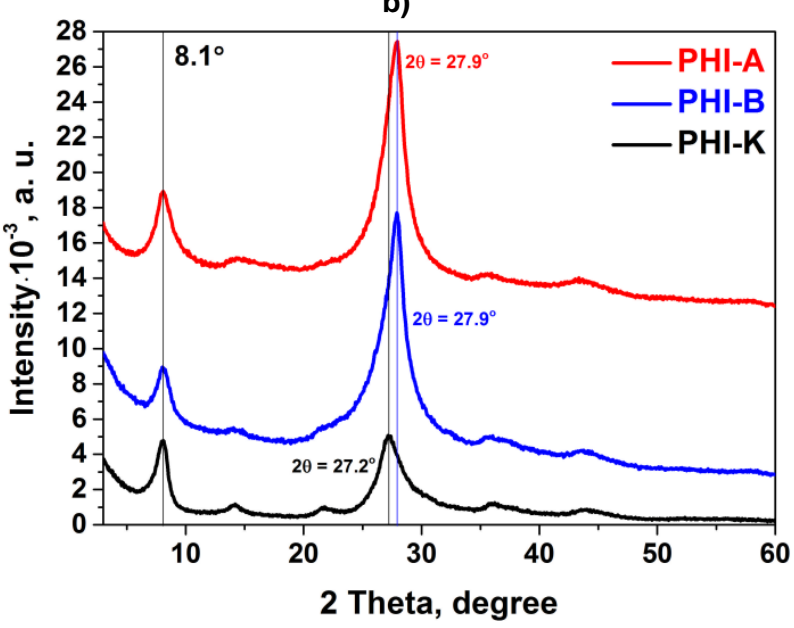

Figure 2. FTIR spectra (a) and PXRD patterns (b) of the original PHI-K, $\mathrm{PHI}-\mathrm{A}$ sample where $\mathrm{K}^{+}$ions are replaced by protons, and $\mathrm{PHI}-\mathrm{B}$, reconstituted $\mathrm{PHI}-\mathrm{K}$ from PHI-A by KOH treatment. Vertical lines in (a) indicate the positions of $1060 \mathrm{~cm}^{-1}$ and $980 \mathrm{~cm}^{-1}$ bands.

FTIR spectra of the acidic PHI-A are compared with that of PHI-K and PHI-B in Figure 2a. The removal of $\mathrm{K}^{+}$ ions is accompanied by the disappearance of the absorption peaks at $1060 \mathrm{~cm}^{-1}$ and $980 \mathrm{~cm}^{-1}$ typically assigned to the asymmetric and symmetric vibrations of $\mathrm{NC}_{2}$ bonds, respectively, in metal- $\mathrm{NC}_{2}$ groups. [15] Therefore, these signals can be used as indicators for a metal presence in the poly(heptazine imide) structure in quick screening tests. Note that the FTIR spectra, in spite of the very different HRTEM and XRD structure, are very similar to those reported by Lotsch et al. [16], i.e. we assume that also our structures are edge-terminated by both amine and cyanamide/carbodiimide groups. This was found to be important for an improved electron transfer towards metal cocatalysts.
In the PXRD patterns of PHI-K, PHI-B and PHI-A (Figure $2 \mathrm{~b}$ ) the peaks at $\sim 8^{\circ}$ and $\sim 27^{\circ}$ are related to the periodicity in between the heptazine columns and their interplanar stacking, respectively, see also the corresponding layer lines in the HRTEM pictures (Figure 1). [12] In the case of potassium-free product, $\mathrm{PHI}-\mathrm{A}$, the peak at $8.1^{\circ}$ is still observed suggesting that the elimination of $\mathrm{K}^{+}$ions preserves the local arrangement of the heptazine columns, and we can assume that the Coulombic character of the deprotonated nitrogen atoms mediated by delocalized protons/hydronium ions prevent transformation into a denser arrangement. The relative intensity and the peak shapes of all scattering peaks are changing as the strongest scatterer, the potassium ion, is removed. In the very end and in agreement with all other data, the structure is however essentially not changing, as shown by the preservation of all main peaks. The only 
deviation is a slight shrinkage breathing of the structure in z-direction for the protonated acid compared to the original $\mathrm{PHI}-\mathrm{K}$, as the main interlayer stacking peak in the PXRD patterns is shifted from $27.2^{\circ}$ to higher 2 theta values of $27.9^{\circ}$, respectively.

Optical and Electronic Properties. The optical properties of $\mathrm{PHI}-\mathrm{K}, \mathrm{PHI}-\mathrm{A}$ and $\mathrm{PHI}-\mathrm{B}$ were studied using UV-visible absorption (Figure 3a) and steady-state photoluminescence (PL) (Figure $3 b$ ) spectroscopies. Two absorption bands are present in the spectra of the materials. One of them (with the edge at $\sim 430-450 \mathrm{~nm}$ ) can be attributed to $\pi \rightarrow \pi^{*}$ transitions, while another one (at

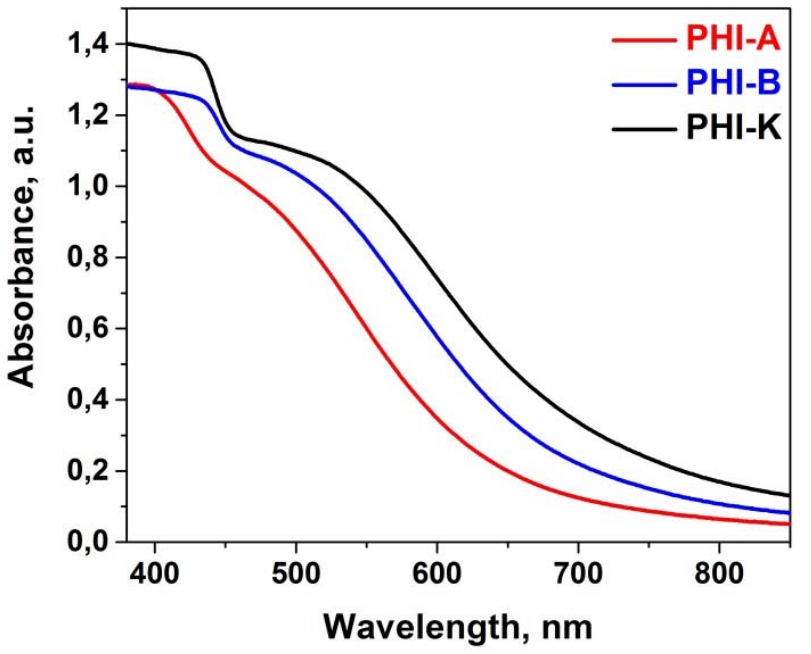

250-600 nm) is speculatively attributed to $n \rightarrow \pi^{*}$ transitions involving electron pairs of the edge nitrogen atoms. [17] Complete removal of $\mathrm{K}^{+}$ions from the structure results in a blue shift of both absorption bands: $\pi \rightarrow \pi^{*}$ absorption band shifts from $450 \mathrm{~nm}$ to $430 \mathrm{~nm}$, while the $n$ $\rightarrow \pi^{*}$ band from $600 \mathrm{~nm}$ to $550 \mathrm{~nm}$ in PHI-A. Optical band gaps calculated from Tauc plots for $\mathrm{PHI}-\mathrm{K}, \mathrm{PHI}-\mathrm{B}$ and $\mathrm{PHI}-$ $\mathrm{A}$ are $1.88 \mathrm{eV}, 2.00 \mathrm{eV}$ and $2.13 \mathrm{eV}$, respectively (Table 1) suggesting an increase of the band gap upon replacing $\mathrm{K}^{+}$ ions with protons. $\mathrm{PHI}-\mathrm{A}$ shows higher photoluminescence compared to $\mathrm{PHI}-\mathrm{K}$ and $\mathrm{PHI}-\mathrm{B}$ (Figure $3 \mathrm{~b}$ ), illustrating the role of the presence of metal cations in the electronic properties of the semiconductor crystals.

a)

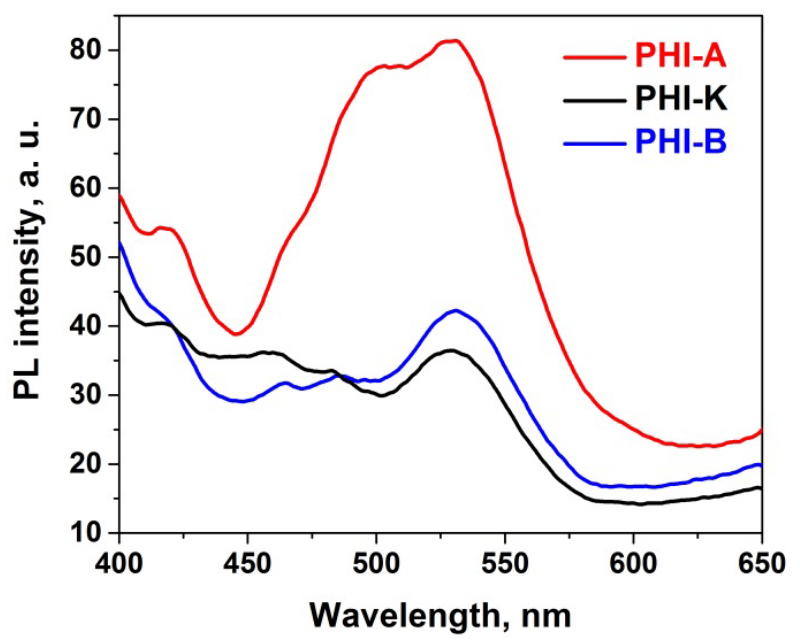

Figure 3. Visible light absorption (a) and steady-state photoluminescence (b) spectra of the products excited at $350 \mathrm{~nm}$.

Also SEM and TEM supports that the morphology of the products PHI-A and PHIK-B is preserved during the ion cycling. Throughout, the morphology of the starting $\mathrm{PHI}-\mathrm{K}$ is kept, represented by oblong needles, ca. $300 \mathrm{~nm}$ in length, growing from joint nucleation seeds (Figure S1).

In order to further reveal the influence of $\mathrm{K}^{+}$ions on the electronic band structures of the resulting polymer salts, electrochemical impedance spectroscopy and MottSchottky analysis were performed (Figure S2). The tested materials show positive slopes of the Mott-Schottky plots revealing a $n$-type semiconductor behavior that enables to consider the values of the found flat-band potentials $\left(E_{F B}\right)$ as approximately corresponding to the conduction band (CB) positions. Potassium ions do not affect the $C B$ position significantly, and the estimated $C B$ potentials of PHI-A, PHI-B and PHI-K products are $-0.05 \mathrm{eV},-0.07 \mathrm{eV}$ and $-0.015 \mathrm{eV}$, respectively. Thus, considering water reduction potential $\left(\varepsilon_{\mathrm{z}^{+} / \mathrm{h}_{\mathrm{g}}}=0 \mathrm{~V}\right)$, PHI-B and the metal- free product $\mathrm{PHI}-\mathrm{A}$ are thermodynamically able to reduce water as it will be demonstrated below. The valence bands (VB) positions can be calculated by subtracting the band gap values (Table 1 ) from the corresponding $C B$ values. They are $2.08 \mathrm{eV}, 1.96 \mathrm{eV}$ and $1.86 \mathrm{eV}$ for PHI-A, PHI-B and $\mathrm{PHI}-\mathrm{K}$, respectively.

Processability. Despite its ionic nature, $\mathrm{PHI}-\mathrm{K}$ is not soluble in water, presumably due to the strong $\pi-\pi$ interactions between the heptazine-based layers. However, it can be dissolved in hot concentrated (98\%) sulfuric acid, similar to polymeric graphitic carbon nitride ( $g-\mathrm{CN})$. [18] Upon neutralization of thus obtained solution, a pale-yellow solid (PHI-SA) precipitates out. The PXRD pattern (Figure $\mathrm{S} 3 \mathrm{a}$ ) of the precipitate is similar to the pattern of melon, [18] while the $\mathrm{C} / \mathrm{N}$ weight ratio (Table S1) of the solid equal to 0.57 further confirms the presence of a weakly condensed melon species with terminal $\mathrm{NH}_{2}$-groups. It is now possible to collect a liquid-state ${ }^{13} \mathrm{C}\left\{{ }^{1} \mathrm{H}\right\} \quad \mathrm{NMR}$ 
spectrum of $\mathrm{PHI}-\mathrm{K}$ in $\mathrm{D}_{2} \mathrm{SO}_{4}$ (Figure $\mathrm{S} 3 \mathrm{~b}$ ), which is similar to the one reported for $\mathrm{g}-\mathrm{CN}$ dissolved in sulfuric acid, reflecting the presence of interconnected heptazine rings. [19] We also attempted to disperse PHI-K in tetraethylammonium hydroxide solution upon prolonged heating, [20] and indeed a colloidal solution was obtained, however the $\mathrm{PHI}$ structure was not preserved, and substantial hydrolysis of $\mathrm{C}-\mathrm{N}$ bonds occurred as evidenced by the significant increase of the oxygen content in the solid from 2.45 to $21.0 \%$, while the $\mathrm{C} / \mathrm{N}$ ratio increased to 0.7 (Table S1).

Alternatively, $\mathrm{PHI}-\mathrm{K}$ can be dispersed without altering significantly its chemical structure, by sequential sonication, centrifugation and freeze-drying steps giving rise to a solid labelled as PHI-D. The separated crystallites tend to agglomerate upon freeze-drying (Figure S4a). $\mathrm{K}^{+}$ ions fully remain in the structure of PHI-D as evidenced by EDX analysis (Table S1), while the optical band gap of PHI-D is increased to $2.64 \mathrm{eV}$ compared to the corresponding value for the original $\mathrm{PHI}-\mathrm{K}$, due to the decrease of the conjugation length and partial exfoliation. Such dispersion preparation procedure results in the increase of the BET surface area of PHI-K from 18 to 49 $\mathrm{m}^{2} / \mathrm{g}$, just by changing the crystallite size.

Ion exchange. Poly(heptazine imide) is not only able to reversibly exchange $\mathrm{K}^{+}$ions by protons, but also can easily and reversibly exchange $\mathrm{K}^{+}$by other metal cations. We replaced $\mathrm{K}^{+}$ions with a variety of $s$ - and $d$-metal cations according to Scheme 2 by agitating $\mathrm{PHI}-\mathrm{K}$ powder in a solution of the desired metal salt, taken in excess relative to the total $\mathrm{K}$ content in the solid (see experimental section for the details). The ion replacement is driven by the difference of metal ion concentrations in the solid structure and in the solution (concentration gradient) as well as the relative affinities and proceeds until a thermodynamic equilibrium is reached. Quantitative replacement of ions can be achieved using a dialysis procedure.

$$
\begin{gathered}
\text { PHI-K } \\
\substack{\mathrm{Li}^{+}, \mathrm{Na}^{+}, \mathrm{Cs}^{+}, \mathrm{Ag}^{+} \\
\mathrm{Mg}^{2+}, \mathrm{Ca}^{2+} \\
\mathrm{Zn}^{2+}, \mathrm{Ni}^{2+}, \mathrm{Co}^{2+}}
\end{gathered}
$$

Scheme 2. lon exchange in $\mathrm{PHI}-\mathrm{K}$ using metals cations studied in current work.

EDX and ICP-AES studies show that $\mathrm{K}^{+}$can be exchanged by $\mathrm{Li}^{+}$or $\mathrm{Na}^{+}$approximately in a 1:1 ratio (Table S3). The amount of $\mathrm{Cs}^{+}$ions that is possible to insert in the structure of $\mathrm{PHI}$ is slightly lower, only about $80 \%$ of $\mathrm{K}^{+}$ion capacity is reached, reflecting the larger ion size of $\mathrm{Cs}^{+}$ (167 pm) compared to $\mathrm{K}^{+}(138 \mathrm{pm})$ and the difficulty of the structure to accommodate the ions. We assume that charge neutrality is reached by protons, then. Conversely, the amount of $\mathrm{Ag}^{+}$ions that can be introduced into $\mathrm{PHI}$ is exceeding that of $\mathrm{K}^{+}$by $18 \%$, illustrating the high affinity of electron-poor $\mathrm{Ag}^{+}$to the electron-rich poly(heptazine imide) even beyond stoichiometry. Among the examined bivalent metal cations, $\mathrm{Ca}^{2+}$ and $\mathrm{Co}^{2+}$ replace $\mathrm{K}^{+}$ions in a 1:2 molar stoichiometry, as expected from charge preservation. The amounts of the introduced $\mathrm{Ni}^{2+}$ and $\mathrm{Mg}^{2+}$ are by $35-40 \%$ smaller than expected. (Table S3). Possibly, this illustrates a lower affinity to the hydrated $\mathrm{Ni}^{2+}$ and $\mathrm{Mg}^{2+}$, while the excess charge is again taken up by protons. Surprisingly, the molar amounts of the inserted $\mathrm{Zn}$ are equal to the original $\mathrm{K}^{+}$content, suggesting that $\mathrm{Zn}$ is introduced as a monovalent cation, presumably as $\mathrm{Zn}(\mathrm{OH})^{+}$.

The introduction of some metal ions, e.g. $\mathrm{Al}, \mathrm{Cu}$ and $\mathrm{Fe}$ using the corresponding metal salts, e.g. $\mathrm{AlCl}_{3} \cdot 6 \mathrm{H}_{2} \mathrm{O}, \mathrm{CuSO}_{4} \cdot 5 \mathrm{H}_{2} \mathrm{O}$ and $\mathrm{FeCl}_{3} \cdot 6 \mathrm{H}_{2} \mathrm{O}$, respectively, is hindered, presumably by the formation of larger solute species, but partly also by the acidity of the added salts (Table S2). Only salts solutions with $\mathrm{pH}$ higher than 4 can be used for metal ion metathesis in $\mathrm{PHI}$; at lower $\mathrm{pH} \mathrm{K}^{+}$ gets quantitatively replaced by protons. The $\mathrm{pH}$ problem can be avoided, however, if the metal salts of weak acids, e.g. acetates are used. Also the ion metathesis in $\mathrm{PHI}$ is found to be reversible. For example, the original $\mathrm{PHI}-\mathrm{K}$ can be reconstituted by agitating $\mathrm{PHI}-\mathrm{Mg}$ in a concentrated $\mathrm{KCl}$ solution.

The chemical structure of poly(heptazine imide) remains unchanged upon ion replacement as suggested by the similarity of the FTIR spectra of PHI-M materials shown in Figure S5. The only exception is PHI-Co, where an additional peaks of $\mathrm{NH}_{2}$-groups at 3565 and $3551 \mathrm{~cm}^{-1}$ appear in the spectrum suggesting that partial protonation of the structure has occurred at the $\mathrm{pH}$ as low as 4.0 (Table S2). PXRD studies (Figure 4) reveal that the scattering peaks, if at all, only change slightly. For the understanding of altered peak intensities and peak shapes it must be said that the entered metal ions are in all cases (except for $\mathrm{Li}$ ) the strongest scattering sources in the system and are potentially not evenly distributed even within the ionic pore space. The latter is illustrated by the almost complete disappearance of the reflection at $2 \theta 8.1^{\circ}$ in the case of the big and heavy $\mathrm{Cs}^{+}$ions, which underlines that these Cs ions are irregularly packed. Only the 
introduction of monovalent $\mathrm{Ag}^{+}$ions into the $\mathrm{PHI}$ structure leads to an apparent significant loss of the order as shown in Figure S6a, that might be due to the need of realizing a

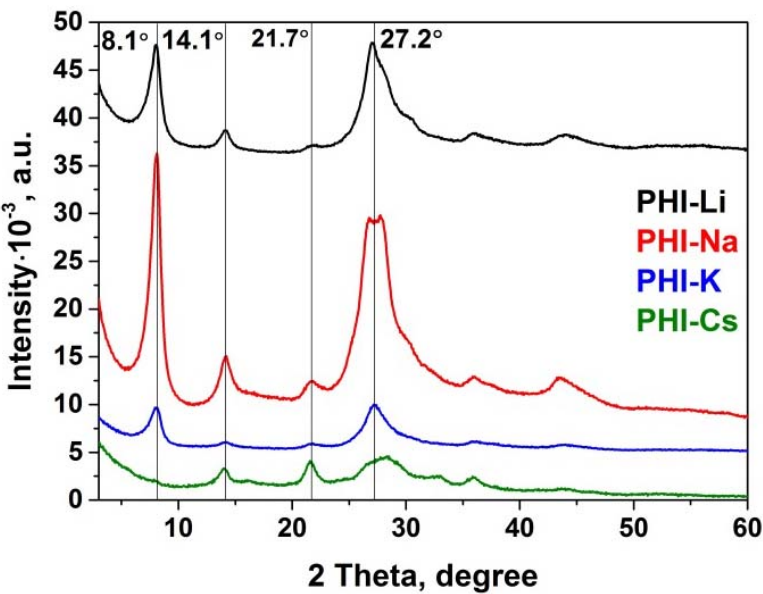

Figure 4. $\mathrm{PXRD}$ patterns of $\mathrm{PHI}-\mathrm{Li}, \mathrm{PHI}-\mathrm{Na}, \mathrm{PHI}-\mathrm{K}, \mathrm{PHI}-\mathrm{Cs}$

When replacing $\mathrm{K}^{+}$with divalent metal ions, a similar behaviour is found (Figure $4 \mathrm{~b}$ ), and only insertion of $\mathrm{Ca}^{2+}$ apparently leads to structural adjustments (Figure S6b). The smaller cation size of $\mathrm{Mg}^{2+}$ (i.r. $76 \mathrm{pm}$ ), $\mathrm{Ni}^{2+}$ (i.r. $69 \mathrm{pm}$ ) and $\mathrm{Zn}^{2+}$ (i.r. $74 \mathrm{pm}$ ) compared to $\mathrm{K}^{+}$(i.r. $138 \mathrm{pm}$ ) leaves the question open if the ions enter partially solvated or even as clusters. The higher uptake of $\mathrm{Zn}^{2+}$ as compared to ideal stoichiometry already indicates that rather interesting species are bound in the pores. In general, introduction of bivalent metal ions is accompanied by a slight shift of the interlayer stacking peak at $2 \theta 27.2^{\circ}$ lower coordination number of $\mathrm{Ag}^{+}$with $\mathrm{N}$-ligands (typically equal to 2 ) than originally provided in $\mathrm{PHI}$.

a)

b)

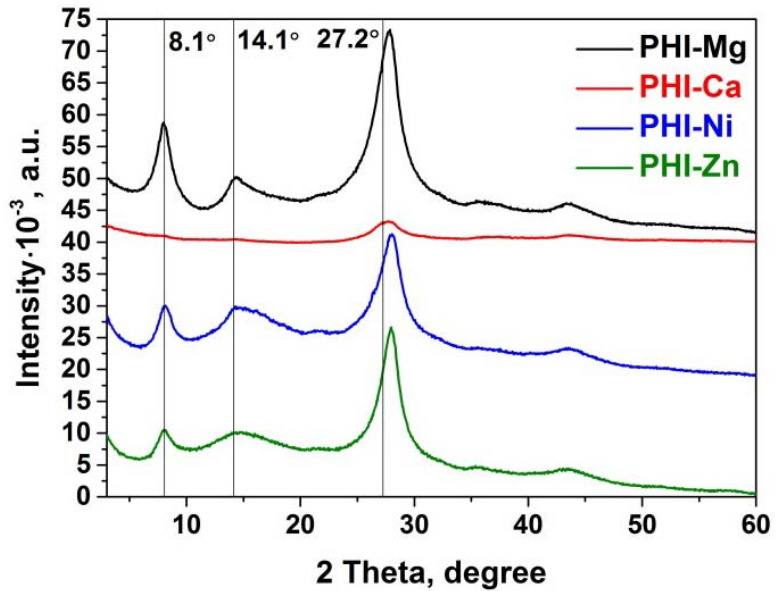

(a) and PHI-Mg, PHI-Ca, PHI-Ni, PHI-Zn (b). to higher values $\left(27.8-28.0^{\circ}\right)$ implying more dense packing of the layers in these materials compared to $\mathrm{PHI}$ $\mathrm{K}$. The replacement of $\mathrm{K}^{+}$with $\mathrm{Co}^{2+}$ again changes the structural arrangement of $\mathrm{PHI}$ more significantly that is probably due to the very strong interactions of $\mathrm{Co}^{2+}$ with $\mathrm{N}$ atoms (Figure S6a). We can nevertheless conclude that the channel system of $\mathrm{PHI}$ is quite flexible and is able to accommodate various metal ions in different ways.

An idealized structure model of the framework systems and the accessible ion channels is provided in Scheme 3.

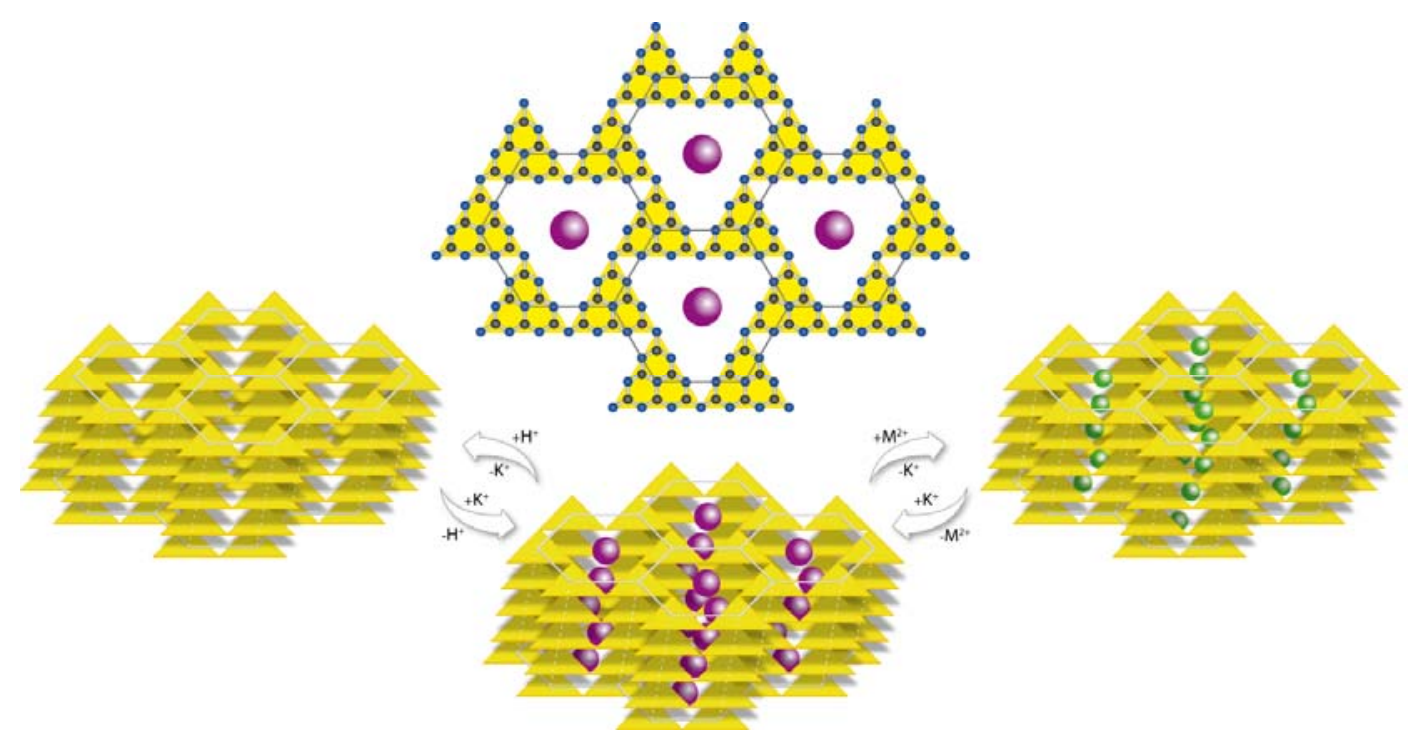

Scheme 3. Suggested idealized poly(heptazine imide) network structure illustrating proton - ion and ion - ion exchange. Yellow triangles represent heptazine units, blue, black, violet and green spheres represent nitrogen, carbon potassium and magnesium atoms/ions, respectively. 
A relevant question for the functionality of these new insertion complexes and their potential catalytic activity is if these structures stay semiconductors after metal exchange, and how the electronic characteristics of the metals can fuse into the joint system. Interestingly, UVvisible absorption spectroscopy and steady-state photoluminescence spectroscopy studies of PHI-M samples showed that the light absorption and emission properties do not change significantly upon variation of the nature of the constituting metal, i.e. the electronic properties of the stacked system of heptazines essentially stays unaffected. The optical band gap values estimated from the corresponding Tauc plots are given in Table 1; they vary between $1.88 \mathrm{eV}$ and $2.00 \mathrm{eV}$. A slight blue-shift of the $\pi \rightarrow \pi^{*}$ absorption band is observed in the cases of $\mathrm{PHI}-\mathrm{Ni}$ and PHI-Co (see Figure S7a) which is likely due to the interactions of $d$ metal orbitals with the $\pi$ system of the polymer, very similar to hypsoporphyrins. [21] In the PL spectra (Figure 5b, Figure S7b), three peaks at 465, 485 and $530 \mathrm{~nm}$ can be observed. Among the studied compounds, PHI-Mg shows the lowest PL yield. This is different from the case of PHI-Ag whose low PL (Figure $\mathrm{S} 7 \mathrm{~b})$ can presumably be explained by the charge carriers' recombination via non-radiative paths, e.g. at a quenching silver superstructure. [22]

The ion exchange has also a minor effect on the morphology and microstructure of poly(heptazine imides), as easily accessed by SEM and illustrated by the similarity of the morphologies of PHI-Mg (Figure S4b) and PHI-K (Figure S1a). The BET surface areas of the materials calculated from the nitrogen sorption isotherms (Figure S8) vary between 10 and $32 \mathrm{~m}^{2} / \mathrm{g}$ and are summarized in Table 1. The electrochemical impedance spectroscopy and MottSchottky analysis revealed that the flat-band potentials of PHI-M vary only slightly with changing metal (Figure S9, Table 1). The estimated $E_{F B}$ values are between $-0.015 \mathrm{~V}$ for $\mathrm{PHI}-\mathrm{K}$ and $-0.163 \mathrm{~V}$ for $\mathrm{PHI}-\mathrm{Co}$ and $\mathrm{PHI}-\mathrm{Ca}$. The analysis is found to be inapplicable for $\mathrm{PHI}-\mathrm{Ni}$ and $\mathrm{PHI}-\mathrm{Ag}$ samples due to co-occurring massive reduction of the metal ions at potentials close to $0 \mathrm{~V}$ (RHE) that changes the nature of the joint electronic system and disturbs the measurements.

Table 1. Optical band gaps, BET surface areas and HER rates* of PHI-M.

\begin{tabular}{|c|c|c|c|c|c|c|c|}
\hline Entry & $\begin{array}{l}\text { Catalyst } \\
\text { name }\end{array}$ & $\begin{array}{l}\text { HER activity, } \\
\mu \mathrm{mol} \cdot \mathrm{h}^{-1}\end{array}$ & $\begin{array}{l}\text { Photo-deposited Pt } \\
\text { amount, wt.\% }\end{array}$ & $\begin{array}{c}\mathrm{E}_{\mathrm{CB}}^{\star *} \\
\mathbf{V}\end{array}$ & $\begin{array}{c}\text { Optical band gap, } \\
\text { eV }\end{array}$ & $\begin{array}{c}E_{V B} \\
V\end{array}$ & $\begin{array}{c}\text { BET surface area, } \\
\mathrm{m}^{2} \cdot \mathrm{g}^{-1}\end{array}$ \\
\hline 1 & PHI-A & 8.48 & 0.61 & -0.05 & 2.13 & 2.08 & 31 \\
\hline 2 & PHI-B & 13.7 & 1.47 & -0.07 & 2.00 & 1.93 & 17 \\
\hline 3 & PHI-D & 17.3 & 0.79 & $\mathrm{n} / \mathrm{a}$ & 2.64 & $\mathrm{n} / \mathrm{a}$ & 49 \\
\hline 4 & PHI-SA & 0 & 0.79 & $\mathrm{n} / \mathrm{a}$ & 2.60 & $\mathrm{n} / \mathrm{a}$ & 43 \\
\hline 5 & PHI-Li & 6.02 & 0.54 & -0.120 & 1.91 & 1.79 & 21 \\
\hline 6 & $\mathrm{PHI}-\mathrm{Na}$ & 10.3 & 3.01 & -0.076 & 1.92 & 1.84 & 21 \\
\hline 7 & PHI-K & 9.70 & 0.94 & -0.015 & 1.88 & 1.86 & 18 \\
\hline 8 & $\mathrm{PHI}-\mathrm{Cs}$ & 10.3 & 1.23 & -0.049 & 1.95 & 1.90 & 15 \\
\hline 9 & PHI-Mg & 25.1 & 1.57 & -0.061 & 1.95 & 1.89 & 17 \\
\hline 10 & $\mathrm{PHI}-\mathrm{Ca}$ & 22.4 & 1.34 & -0.163 & 1.92 & 1.76 & 21 \\
\hline 11 & $\mathrm{PHI}-\mathrm{Zn}$ & 9.57 & 1.91 & -0.140 & 1.95 & 1.81 & 27 \\
\hline 12 & PHI-Co & 2.87 & 1.71 & -0.163 & 2.00 & 1.84 & 32 \\
\hline 13 & $\mathrm{PHI}-\mathrm{Ni}$ & 1.92 & 0.66 & - & 2.00 & - & 31 \\
\hline 14 & PHI-Ag & 0 & 5.16 & - & 1.90 & - & 10 \\
\hline
\end{tabular}

n/a: not analyzed.

*HER was conducted using $20 \mathrm{mg}$ of the catalysts, TEOA as a sacrificial agent, in situ photodeposited Pt as a co-catalyst and $50 \mathrm{~W}$ white LED as an irradiation source.

${ }^{* *}$ from Mott-Schottky analysis.

Photochemical water reduction. As a quick cross-test of preserved semiconductor functionality and long range charge transport and motivated by the fact that all materials give a rather nice and broad adsorption of the visible range of light, all the prepared materials were tested in visible light driven hydrogen evolution reaction (HER) 
using triethanolamine (TEOA) as a sacrificial agent and photodeposited $\mathrm{Pt}$ as a co-catalyst. The experimental details can be found in the Supporting Information, and the results of these studies are summarized in Table 1. All PHI-M except for PHI-Ag are found to be active photocatalysts for hydrogen production, while the activity depends on the nature of the $M$ in the structure. The activities of alkali metal containing PHI-M ( $\mathrm{M}=\mathrm{Na}, \mathrm{K}, \mathrm{Cs})$ are close to each other and are about $10.0 \mu \mathrm{mol} \mathrm{H}_{2} / \mathrm{hour}$. A somewhat lower activity of $6.0 \mu \mathrm{mol} \mathrm{H}_{2} /$ hour is found for $\mathrm{PHI}-\mathrm{Li}$ and is presumably due to the lower Pt loading of 0.54 wt.\%. Earth-alkali metal containing PHI-M show a significantly improved activity and a more than twice higher hydrogen evolution rates. The highest activity of $25.1 \mu \mathrm{mol}$ $\mathrm{H}_{2} /$ hour is measured for PHI-Mg. Magnesium itself should not participate in water reduction processes, since the

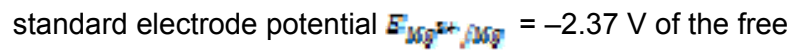
ions lies way below water reduction potential $E_{\mathrm{zs}^{+} / \mathrm{h}_{\mathrm{g}}}=0$ $\mathrm{V}$ and the LUMO levels. Interestingly, it is also in the biological porphyrine system where $\mathrm{Mg}^{2+}$ ions are stabilizing the organic system towards the highly efficient light harvesting chlorophyll moieties, while diverse packing of the dyes modifies their energy positions. In those terms, our PHI-Mg nicely follows the natural original. Except for $\mathrm{PHI}-\mathrm{Zn}$, a redox inert metal with a filled $d^{10}$-configuration, whose activity is similar to that of $\mathrm{PHI}-\mathrm{K}, d$-elements derived products are much less active. The drop of activity is assigned to the possible ligand-to-metal charge transfers that result in transitions which are too low in energy to participate in the overall process of water photoreduction. This however might be instrumentalized for other, metalcenter based photocatalytic reactions.

\section{Conclusions}

The thermal condensation of substituted 1,2,4-triazoles in eutectic salt melts leads to highly crystalline potassium (heptazine imides, PHI ). The local structure of the crystals is characterized by regular ion channels arranged within a semiconducting matrix formed by tightly packed heptazine units. It was shown that the potassium ions in $\mathrm{PHI}$ can be quantitatively extracted using diluted acid solutions and/or exchanged to other metal ions while the crystal structure and habitus is essentially preserved. Various alkali, earthalkali or transition metals could be incorporated, while conductivity, optical and electronic properties of thus obtained $\mathrm{PHI}$ salts was shown to weakly depend on the nature of the introduced cations. Photochemical water reduction i.e. hydrogen production was applied as a model reaction to illustrate the catalytic usefulness of the resulting systems, and similar to the natural chlorophyll, the magnesium containing system turned out to be most efficient, which could be related to an improved charge separation and localization efficiency. Owing to their unique structural and electronic properties, $\mathrm{PHI}$ salts are potentially interesting as solid state ion conductors for battery applications. Another promising application field for $\mathrm{PHI}-\mathrm{M}$ is catalysis. Here, specific transition-metal ions can be incorporated into $\mathrm{PHI}$ framework to catalyse target reactions or promote desired selectivity. Considering the structural similarities between $\mathrm{PHI}$ and porphyrins, one can easily imagine biomimetic catalytic applications.

Keywords: carbon nitride, poly(heptazine imide), porous solid polyelectrolytes, organic zeolites

\section{References}

1) K. Mizushima, P. C. Jones, P. J. Wiseman, J. B. Goodenough, Mater. Res. Bull., 1980, 15, 783.

2) M. G. S. R. Thomas, P. G. Bruce, J. B. Goodenough, Solid State lonics, 1985, 17, 13.

3) H.P. Ma, B.L. Liu, B. Li, L.M. Zhang, Y.G. Li, H.Q. Tan, H.Y. Zang,; G.S. Zhu, GS : J.Am.Chem.Soc. 2016, 138, 5897-5903

4) X. Wang, K. Maeda, A. Thomas, K. Takanabe, G. Xin, J. M. Carlsson, K. Domen, M. Antonietti, Nat. Mater., 2009, 8(1), 76-80.

5) K. Maeda, X. Wang, Y. Nishihara, D. Lu, M. Antonietti, K. Domen, J. Phys. Chem. C, 2009, 113(12), 4940-4947.

6) E. Wirnhier, M. Döblinger, D. Gunzelmann, J. Senker, B. V. Lotsch, W. Schnick, Chem.-Eur. J., 2011, 17(11), 3213-3221.

7) M. K. Bhunia, K. Yamauchi, K. Takanabe. Angew. Chem. Int. Ed. 2014, 53, 11001-11005.

8) Y. Wang, X. Wang, M. Antonietti, Angew. Chem. Int. Ed. 2012, 51, 68-89.

9) S. Yin et al., Catal. Sci. Technol., 2015, 5, 5048-5061.

10) S. Cao, J. Low, J. Yu, M. Jaroniec. Adv. Mater. 2015, 27, 21502176.

11) S. Y. Chong, J. T. A. Jones, Y. Z. Khimyak, A. I. Cooper, A. Thomas, M. Antonietti, M. J. Bojdys, J. Mater. Chem. A. 2013, 1, 4, 1102-1107.

12) D. Dontsova, S. Pronkin, M. Wehle, Z. Chen, C. Fettkenhauer, G. Clavel, M. Antonietti, Chem. Mater., 2015, 27 (15), 5170-5179.

13) C. F. J. Faul, M. Antonietti, Adv. Mater. 2003, 15, 6, 673-683.

14) D. Dontsova, C. Fettkenhauer, V. Papaefthimiou, J. Schmidt, M. Antonietti, Chem. Mater. 2016, 28, 772-778.

15) D.C. Bradley, M.H. Gitlitz, J. Chem. Soc. (A), 1969, $980-984$

16) V.W.H. Lau, I. Moudrakovski, T. Botari, S. Weinberger, M.B. Mesch, V. Duppel, J. Senker, V. Blum, B.V. Lotsch, Nature Comm DOI: 10.1038/ncomms 12165

17) Jorge, A. B.; Martin, D. J.; Dhanoa, M. T. S.; Rahman, A. S.; Makwana, N.; Tang, J.; Sella, A.; Corà, F.; Firth, S.; Darr, J. A. McMillan, P. F. J. Phys. Chem. C 2013, 117, 7178-7185.

18) Z. Zhou, J. Wang, J. Yu, Y. Shen, Y. Li, A. Li, S. Liu, Y. Zhang. J. Am. Chem. Soc., 2015, 137, 2179-2182.

19) B. V. Lotsch. From Molecular Building Blocks to Condensed Carbon Nitride Networks: Structure and Reactivity, 2006, p.294.

20) Y. V. Panasiuk, A. E. Raevskaya, O. L. Stroyuk, P. M. Lytvyn, S. Ya. Kuchmiy. RSC Adv., 2015, 5, 46843-46849.

21) D. F. Marsh, L. M. Mink, J. Chem. Ed., 1996, 73 (12), 1181.

22) M. Shalom et al., J. Am. Chem. Soc., 2013, 135(19), 7118-7121. 


\section{Supporting information}

\section{Experimental section}

Potassium poly(heptazine)imide (PHI-K) was prepared according to the method reported previously.[11]

PHI-A synthesis. A mixture of PHI-K (300 mg) and $\mathrm{HCl}\left(15 \mathrm{~mL}, 0.1 \mathrm{~mol} \cdot \mathrm{L}^{-1}\right)$ was maintained in sonication bath for 2 hours. Suspension was stirred at room temperature for 48 hours and centrifuged (5500 $\mathrm{min}^{-1}, 15 \mathrm{~min}$ ). Precipitate was separated, washed with distilled water until $\mathrm{pH}=7$ and dried in vacuum giving $202 \mathrm{mg}$ of bright orange solid.

PHI-B synthesis. A mixture of PHI-K (300 mg) and $\mathrm{KOH}$ solution $\left(15 \mathrm{~mL}, 0.1 \mathrm{~mol} \cdot \mathrm{L}^{-1}\right)$ was maintained in sonication bath for 2 hours. Suspension was stirred at room temperature for 48 hours and centrifuged (5500 $\mathrm{min}^{-1}, 15 \mathrm{~min}$ ). Precipitate was separated, washed with distilled water until $\mathrm{pH}=7$ and dried in vacuum giving $211 \mathrm{mg}$ of dark orange solid.

PHI-SA synthesis. A mixture of PHI-K (560 mg) and $\mathrm{H}_{2} \mathrm{SO}_{4}(5 \mathrm{~mL}, 98 \%)$ was stirred at $100{ }^{\circ} \mathrm{C}$ for 4 hours and cooled to room temperature. A concentrated $\mathrm{KOH}(4.7 \mathrm{~g})$ solution was added slowly to the reaction mixture, then the $\mathrm{pH}$ of the solution was adjusted to 7 with $\mathrm{K}_{2} \mathrm{CO}_{3}(1 \mathrm{M})$ solution. Precipitate was filtered and washed thoroughly with water giving $148 \mathrm{mg}$ of pale-yellow solid.

PHI-D synthesis. PHI-K $(0.5 \mathrm{~g})$ and water $(50 \mathrm{~mL})$ was sonicated in the ultrasonic bath for $8 \mathrm{~h}$, precipitate was separated by centrifugation $\left(2000 \mathrm{~min}^{-1}, 10 \mathrm{~min}\right)$. The remaining dispersion was frozen in liquid nitrogen and water sublimed in vacuum giving 'fluffy' yellow powder. The solid after centrifugation was again subjected to the abovementioned procedure, totally 8 times. After each cycle 10-25 mg of the PHI-D were obtained.

PHI-Li, PHI-Na, PHI-Cs, PHI-Mg, PHI-Ca, PHI-Ni, PHI-Co and PHI-Zn synthesis. A membrane tubing (Spectra/Por Dialysis Tubing, MWCO 1000) was filled with a suspension of PHI-K (see Table S1) in water (5-10 mL). The tubing was closed tightly from both sides using clips and placed into a beaker with stir bar. Appropriate salt solution (the salt mass and water volume taken for solution preparation are listed in the Table S2) was added and suspension was subjected for dialysis for 7 days with constant stirring. Every 24 hours salt solution was changed with a fresh portion. The solid was separated by centrifugation, washed with distilled water $(3 \times 1.5 \mathrm{~mL})$ and dried in vacuum giving the product of giving mass.

PHI-Ag synthesis. A flask (5 mL) was charged with $\mathrm{PHI}-\mathrm{K}, \mathrm{AgNO}_{3}$ solution (Table S1) and stir bar. The flask was covered with black tissue to avoid Ag $^{+}$reduction. Suspension was stirred at room temperature for 6 days. Every 24 hours salt solution was changed with new portion. The precipitate was separated by centrifugation, washed with distilled water $(3 \times 1.5 \mathrm{~mL})$ and dried in vacuum. 

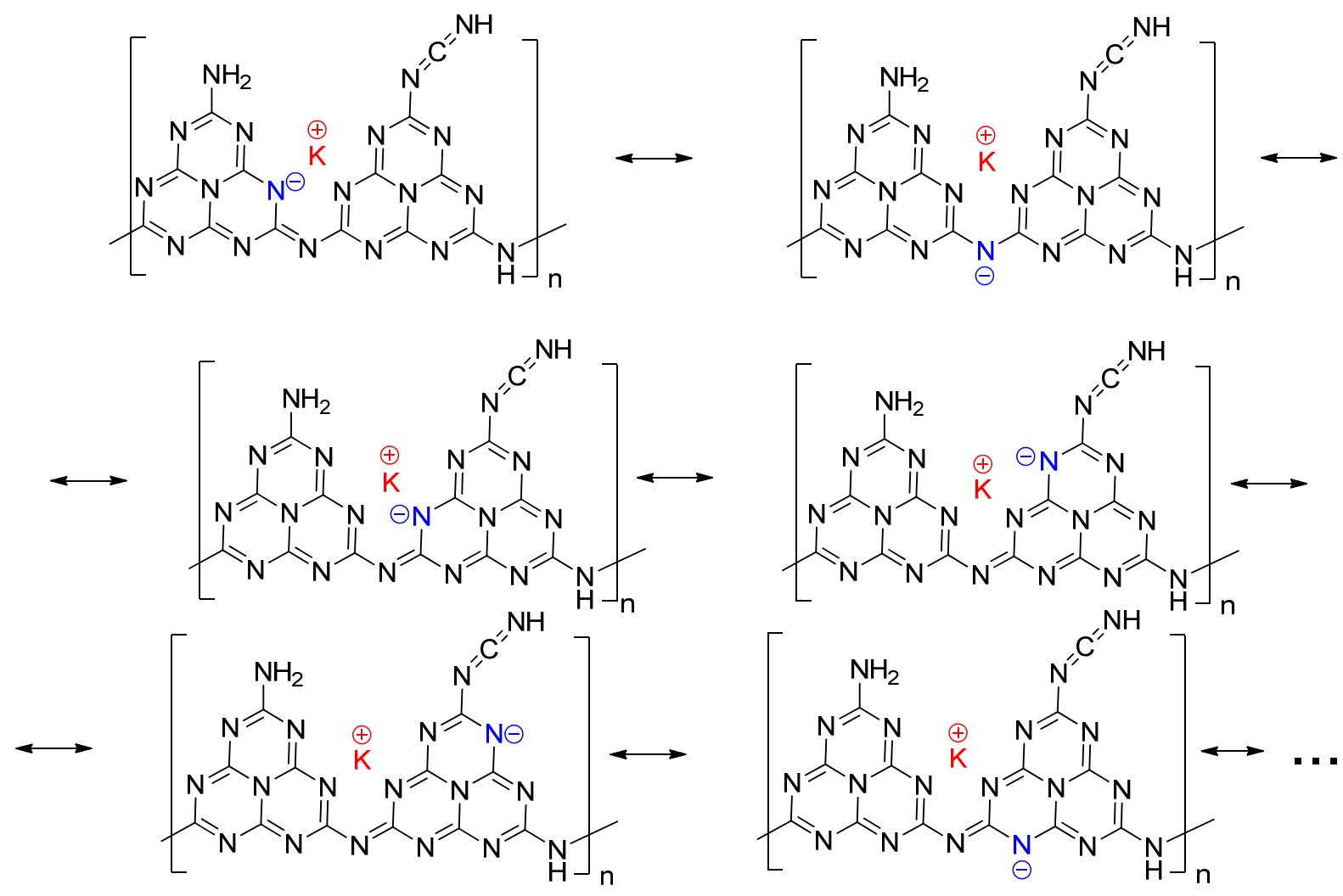

Scheme S1. Resonance structures of PHI-K.

Table S1. Elemental composition of the products obtained from potassium poly(heptazine imide) by acid or base treatment, according to EDS and EA (H content) data.

\begin{tabular}{|c|c|c|c|c|c|c|c|c|c|}
\hline Entry & Conditions of PHI-K treatment & $\begin{array}{l}\text { Product } \\
\text { name }\end{array}$ & $\begin{array}{c}\text { C, } \\
\text { wt.\% }\end{array}$ & $\begin{array}{c}\mathrm{N}, \\
\text { wt. } \%\end{array}$ & $\begin{array}{c}\text { H, } \\
\text { wt.\% }\end{array}$ & $\mathrm{C} / \mathrm{N}$ & $\begin{array}{c}\mathrm{K}, \\
\text { wt. } \%\end{array}$ & $\begin{array}{c}0, \\
\text { wt. } \%\end{array}$ & $\begin{array}{c}\mathrm{Cl}, \\
\text { wt.\% }\end{array}$ \\
\hline 1 & & $\begin{array}{c}\text { PHI-K, } \\
\text { theoretical } \\
\mathrm{C}_{12} \mathrm{~N}_{18} \mathrm{H}_{5} \mathrm{~K}\end{array}$ & 32.73 & 57.25 & 1.14 & 0.572 & 8.88 & - & - \\
\hline 2 & original product & PHI-K & 32.67 & 52.69 & 2.450 & 0.62 & 9.63 & 2.45 & 0.11 \\
\hline 3 & $0.1 \mathrm{M} \mathrm{HCl}$ solution & PHI-A & 36.10 & 58.22 & 3.184 & 0.62 & 0.01 & 2.46 & 0.03 \\
\hline 4 & $\begin{array}{l}\text { PHI-A, treated with } 2.0 \mathrm{M} \quad \mathrm{KOH} \\
\text { solution }\end{array}$ & PHI-B & 32.79 & 52.90 & 2.599 & 0.62 & 8.92 & 2.79 & 0.00 \\
\hline 5 & & Melon & 35.83 & 62.67 & 1.50 & 0.57 & - & - & - \\
\hline 6 & $\begin{array}{l}\text { Dissolution in concentrated } \mathrm{H}_{2} \mathrm{SO}_{4} \\
\text { followed by precipitation with } \mathrm{K}_{2} \mathrm{CO}_{3}\end{array}$ & PHI-SA & 31.01 & 54.41 & 3.77 & 0.57 & 0.04 & 10.77 & 0.00 \\
\hline 7 & \begin{tabular}{|l} 
Sonication, centrifugation and \\
freeze-drying
\end{tabular} & PHI-D & 30.14 & 42.45 & 2.13 & 0.71 & 9.75 & 15.42 & 0.11 \\
\hline 8 & $\begin{array}{l}\text { Heating in the solution of } \mathrm{N}(\mathrm{Et})_{4} \mathrm{OH} \\
\text { at } 100^{\circ} \mathrm{C} \text { for } 8 \text { hours }\end{array}$ & PHI-OH & 29.17 & 41.68 & 3.217 & 0.70 & 4.52 & 20.99 & 0.42 \\
\hline
\end{tabular}


Table S2. Details on the synthesis of PHI-M.

\begin{tabular}{|l|l|l|l|l|l|l|l|l|}
\hline Entry & $\begin{array}{l}\text { Product } \\
\text { name }\end{array}$ & $\begin{array}{l}\text { PHI-K } \\
\text { amount } \\
\text { taken, } \\
\text { mg }\end{array}$ & $\begin{array}{l}\text { Metal } \\
\text { cation }\end{array}$ & Salt used & $\begin{array}{l}\text { Amount } \\
\text { of the } \\
\text { salt, g }\end{array}$ & $\begin{array}{l}\text { Water, } \\
\mathrm{mL}\end{array}$ & $\begin{array}{l}\text { Solution } \\
\mathrm{pH}\end{array}$ & $\begin{array}{l}\text { Product } \\
\text { yield, mg }\end{array}$ \\
\hline 1 & PHI-Li & 120 & $\mathrm{Li}^{+}$ & $\mathrm{LiCl}$ & 20 & 80 & 6.6 & 102 \\
\hline 2 & PHI-Na & 150 & $\mathrm{Na}^{+}$ & $\mathrm{NaCl}$ & 10 & 30 & 7.1 & 143 \\
\hline 3 & PHI-Cs & 150 & $\mathrm{Cs}^{+}$ & $\mathrm{CsCl}$ & 1 & 2 & 8.0 & 186 \\
\hline 1 & PHI-Mg & 250 & $\mathrm{Mg}^{2+}$ & $\mathrm{MgCl}_{2} \cdot 6 \mathrm{H}_{2} \mathrm{O}$ & 50 & 100 & 4.7 & 227 \\
\hline 3 & PHI-Ca & 120 & $\mathrm{Ca}^{2+}$ & $\mathrm{CaCl}_{2} \cdot 2 \mathrm{H}_{2} \mathrm{O}$ & 20 & 70 & 4.2 & 114 \\
\hline 8 & PHI-Co & 150 & $\mathrm{Co}^{2+}$ & $\mathrm{CoCl}_{2} \cdot 6 \mathrm{H}_{2} \mathrm{O}$ & 0.5 & 1 & 4.0 & 156 \\
\hline 7 & PHI-Ni & 150 & $\mathrm{Ni}^{2+}$ & $\mathrm{NiCl}_{2} \cdot 6 \mathrm{H}_{2} \mathrm{O}$ & 0.5 & 1 & 4.3 & 150 \\
\hline 5 & $\mathrm{PHI}-\mathrm{Zn}$ & 150 & $\mathrm{Zn}^{2+}$ & $\mathrm{ZnCl}_{2}$ & 10 & 40 & 4.6 & 158 \\
\hline 2 & $\mathrm{PHI}-\mathrm{Ag}$ & 120 & $\mathrm{Ag}^{+}$ & $\mathrm{AgNO}_{3}$ & 0.120 & 1.5 & 5.2 & 201 \\
\hline 9 & PHI-Al & 150 & $\mathrm{Al}^{3+}$ & $\mathrm{AlCl}_{3} \cdot 6 \mathrm{H}_{2} \mathrm{O}$ & 10 & 30 & 1.0 & $\begin{array}{l}\text { not } \\
\text { determined }\end{array}$ \\
\hline 10 & PHI-Cu & 150 & $\mathrm{Cu}^{2+}$ & $\mathrm{CuSO}_{4} \cdot 5 \mathrm{H}_{2} \mathrm{O}$ & 10 & 30 & 3.3 & $\begin{array}{l}\text { not } \\
\text { determined }\end{array}$ \\
\hline 11 & PHI-Fe & 150 & $\mathrm{Fe}^{3+}$ & $\mathrm{FeCl}_{3} \cdot 6 \mathrm{H}_{2} \mathrm{O}$ & 10 & 30 & 0.5 & $\begin{array}{l}\text { not } \\
\text { determined }\end{array}$ \\
\hline
\end{tabular}

a)

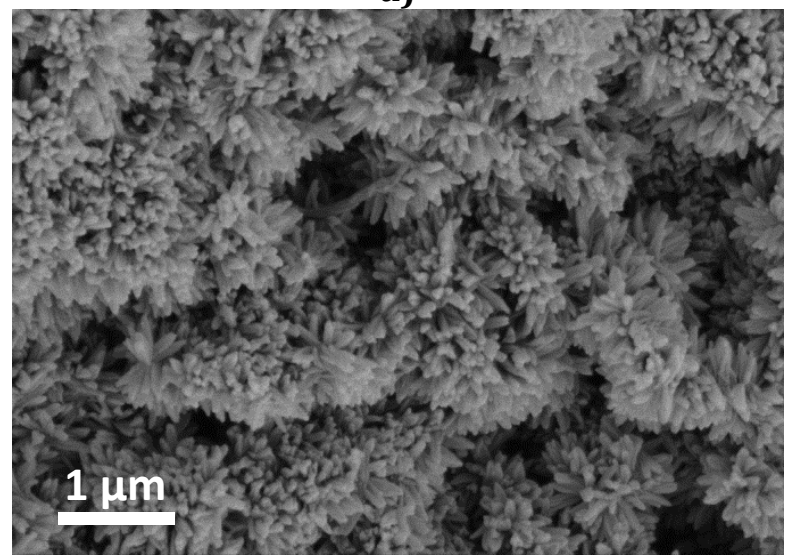

c)

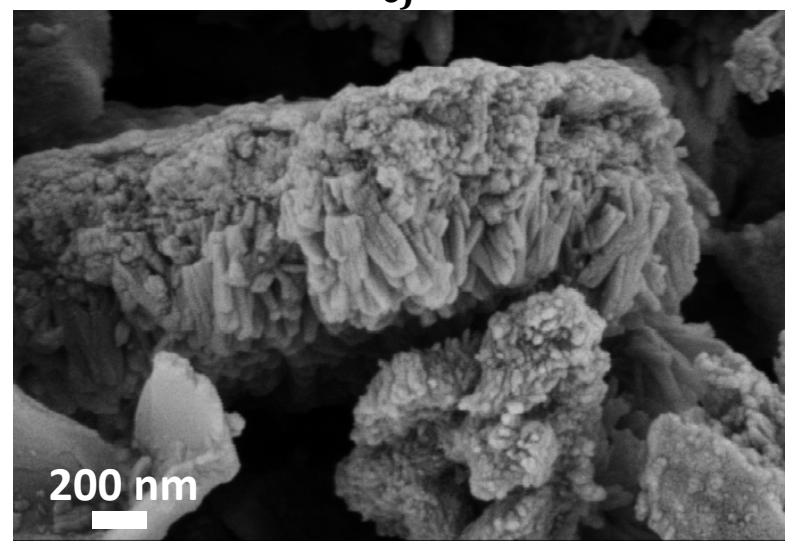

b)

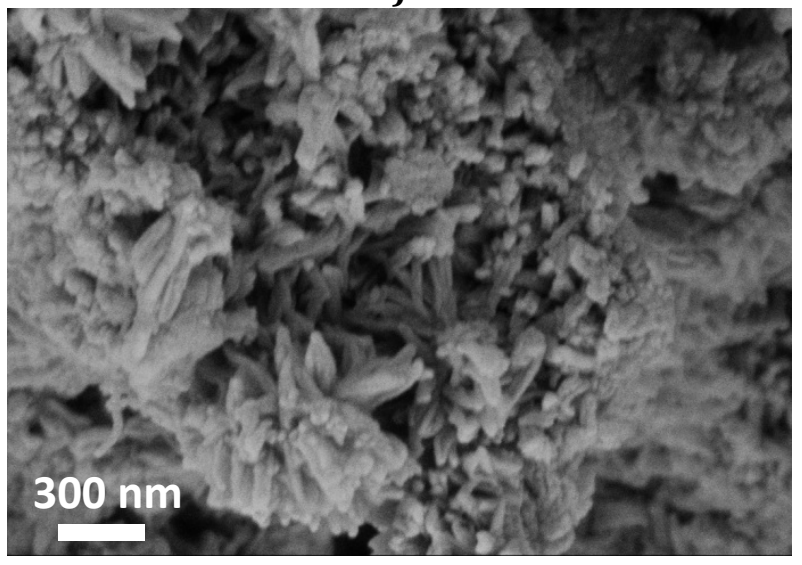

Figure S1. SEM images of PHI-K (a), PHI-A (b), and PHI-B (c) 


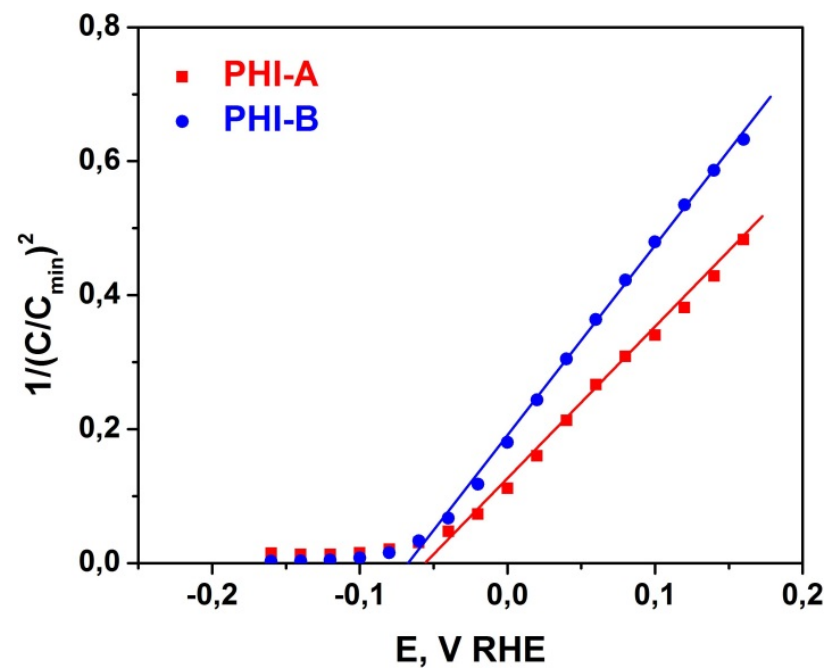

Figure S2. Mott-Schottky plots of PHI-A and PHI-B materials. 
a)

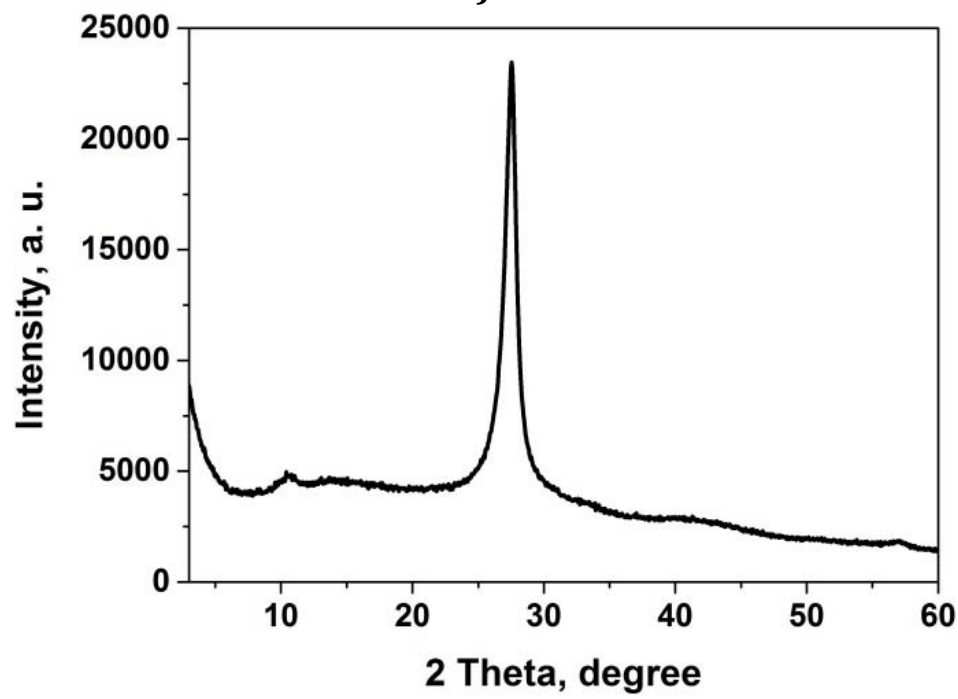

b)

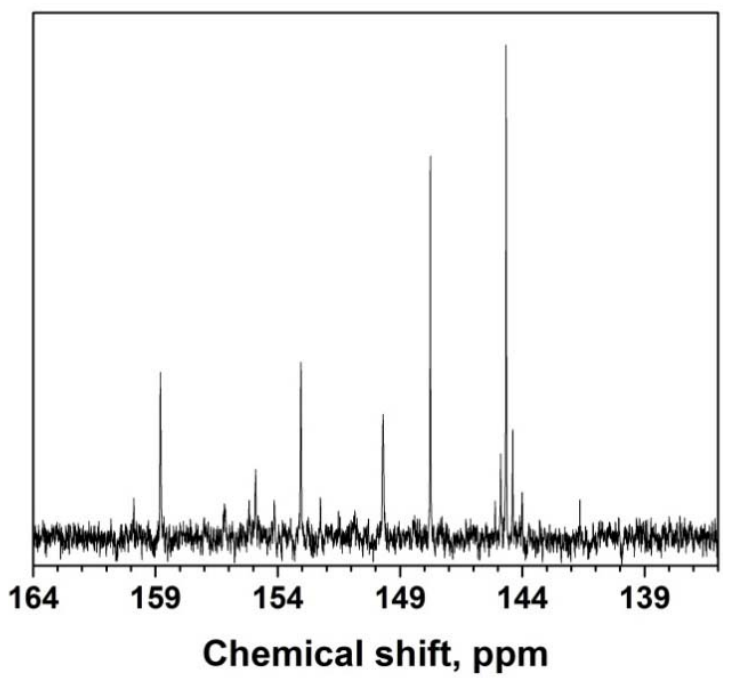

Figure S3. PXRD pattern of PHI-SA (a) and ${ }^{13} \mathrm{C}\left\{{ }^{1} \mathrm{H}\right\}$ NMR spectrum of $\mathrm{PHI}-\mathrm{K}_{\text {in }} \mathrm{D}_{2} \mathrm{SO}_{4}$ (b).

a)

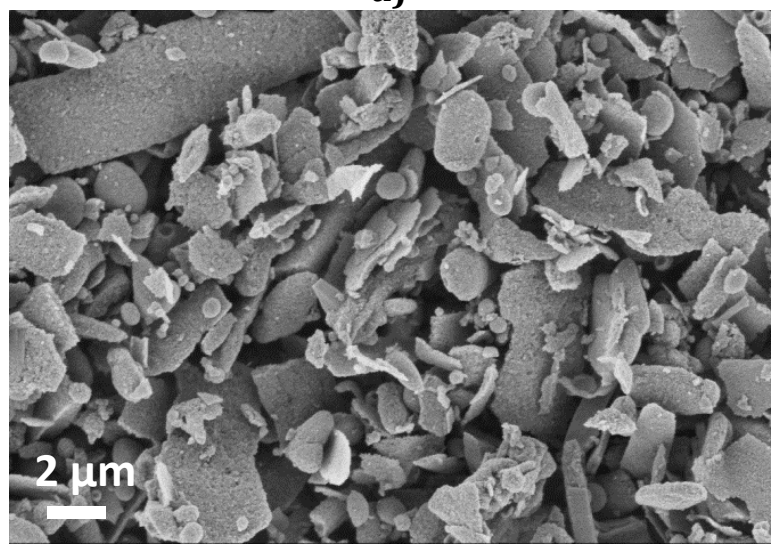

b)

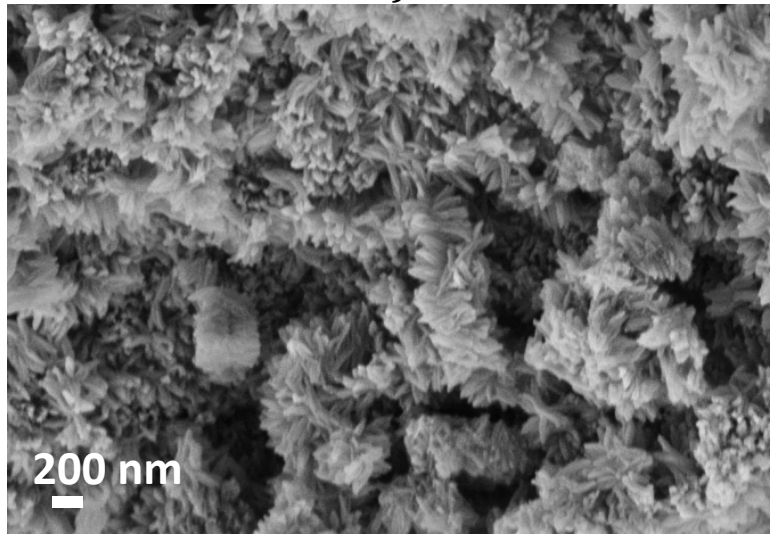

Figure S4. SEM images of PHI-D (a) and PHI-Mg (b).

Table S3. Elemental composition of the PHI-M materials based on elemental (H), EDS and ICP (Li) analyses.

\begin{tabular}{|c|c|c|c|c|c|c|c|c|c|c|}
\hline Entry & $\begin{array}{c}\text { Product } \\
\text { name }\end{array}$ & $\begin{array}{c}\text { C, } \\
\text { wt.\% }\end{array}$ & N, wt.\% & $\begin{array}{c}\mathbf{H}, \\
\text { wt.\% }\end{array}$ & $\mathbf{C / N}$ & $\begin{array}{c}\text { M, } \\
\text { wt. \% }\end{array}$ & $\begin{array}{c}\text { M, } \\
\text { moles }\end{array}$ & 0, wt.\% & $\begin{array}{c}\text { Cl, } \\
\text { wt.\% }\end{array}$ & $\begin{array}{c}\text { Cl, } \\
\text { moles }\end{array}$ \\
\hline 1 & PHI-Li & 33.5 & 54.4 & 3.02 & 0.615 & $2.22^{\mathrm{a}}$ & 0.322 & 6.79 & 0.04 & 0.001 \\
\hline 2 & PHI-Na & 32.9 & 51.9 & 2.91 & 0.634 & 6.60 & 0.287 & 5.51 & 0.14 & 0.004 \\
\hline 3 & PHI-K & 31.8 & 51.3 & 2.45 & 0.620 & 11.88 & 0.305 & 2.45 & 0.02 & 0 \\
\hline 4 & PHI-Cs & 25.2 & 39.6 & 1.93 & 0.636 & 32.53 & 0.245 & 0.61 & 0.20 & 0.006 \\
\hline 5 & PHI-Mg & 32.7 & 53.0 & 3.22 & 0.617 & 2.70 & 0.111 & 7.10 & 1.23 & 0.035 \\
\hline 6 & PHI-Ca & 31.6 & 51.2 & 2.92 & 0.617 & 6.72 & 0.168 & 7.58 & 0.03 & 0.001 \\
\hline 7 & PHI-Co & 30.8 & 48.6 & 2.84 & 0.634 & 9.51 & 0.161 & 6.66 & 1.56 & 0.044 \\
\hline
\end{tabular}




\begin{tabular}{|c|c|c|c|c|c|c|c|c|c|c|}
\hline 8 & PHI-Ni & 32.9 & 52.2 & 2.83 & 0.630 & 6.06 & 0.103 & 5.87 & 0.13 & 0.004 \\
\hline 9 & PHI-Zn & 26.7 & 42.2 & 2.38 & 0.634 & 19.07 & 0.292 & 7.97 & 1.65 & 0.046 \\
\hline 10 & PHI-Ag & 19.3 & 32.9 & 1.08 & 0.586 & 38.89 & 0.360 & 7.94 & 0.00 & 0 \\
\hline
\end{tabular}

a data from ICP analysis.

a)

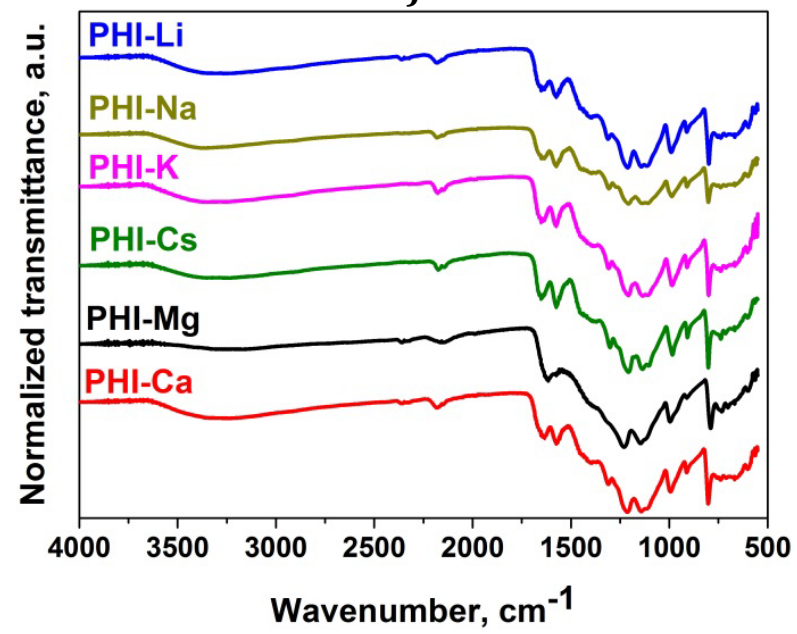

Figure S5. FTIR -ATR spectra of PHI-M solids.

a)

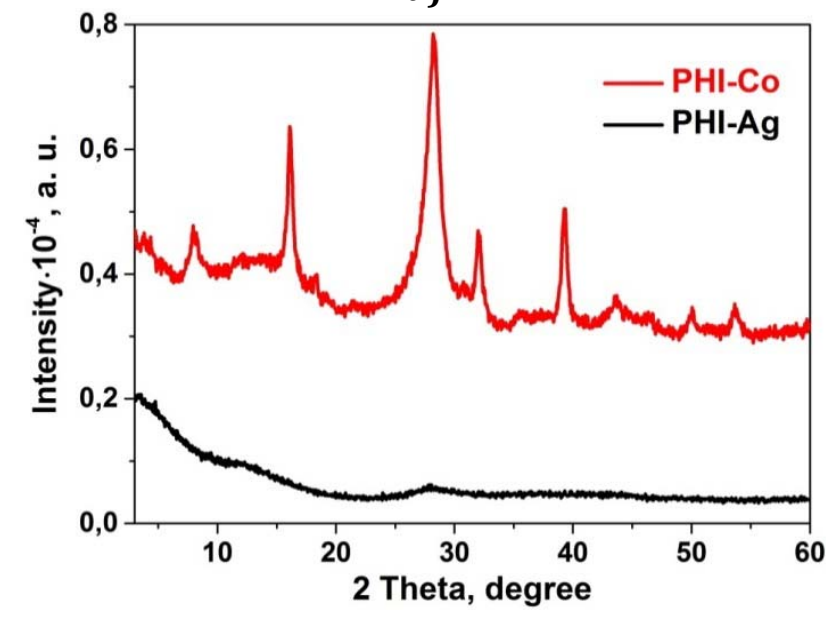

b)

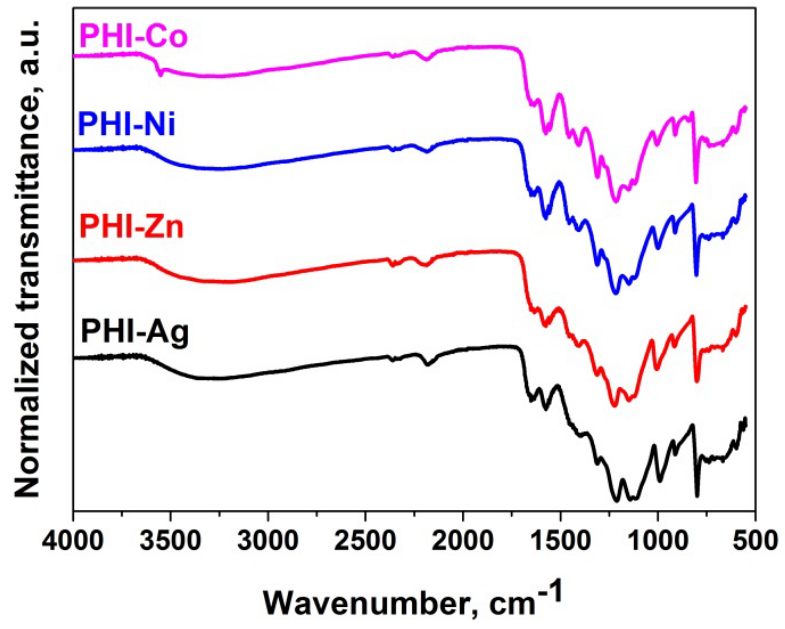

Wavenumber, $\mathrm{cm}^{-1}$

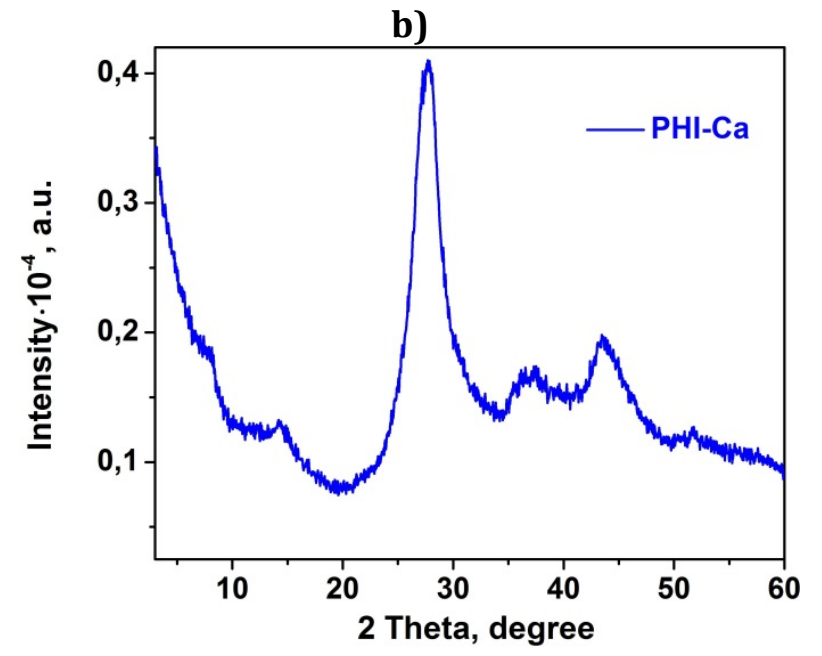

Figure S6. PXRD pattern of PHI-Co and PHI-Ag (a) and PHI-Ca (b). 
a)

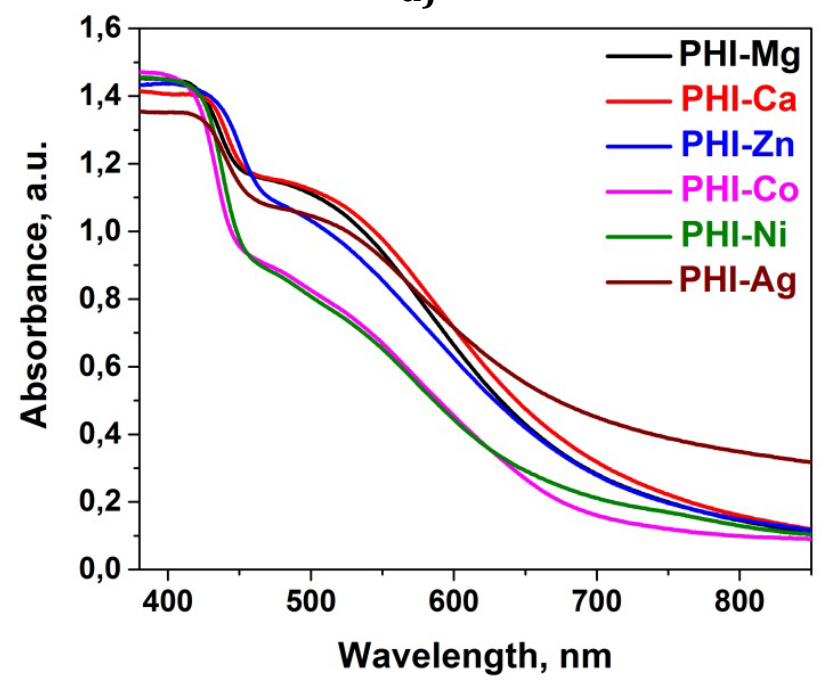

b)

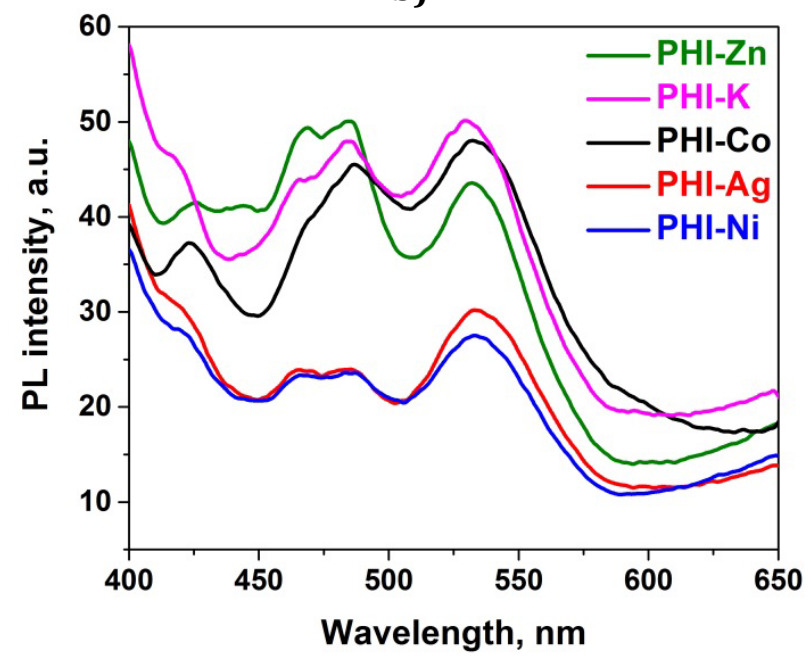

Figure S7. UV-vis absorption (a) and steady-state photoluminescence (b) spectra of PHI-M materials.

a)

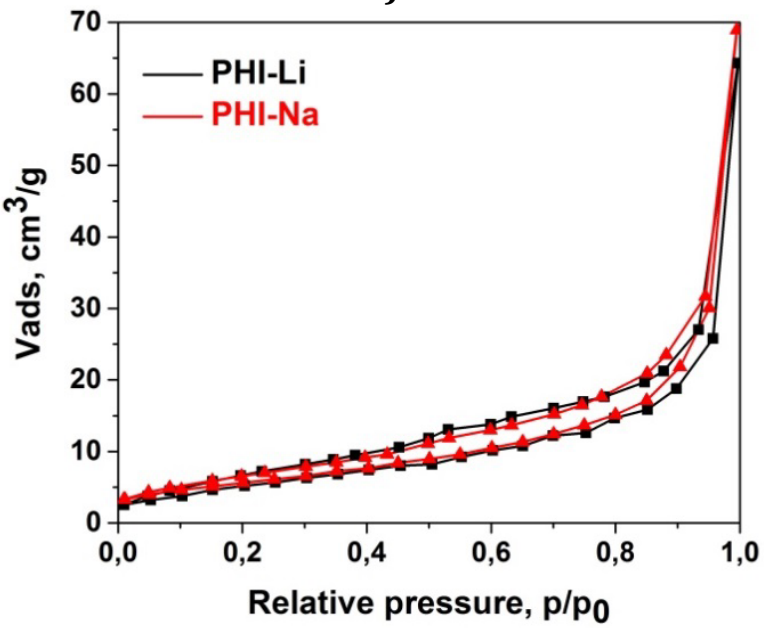

c)

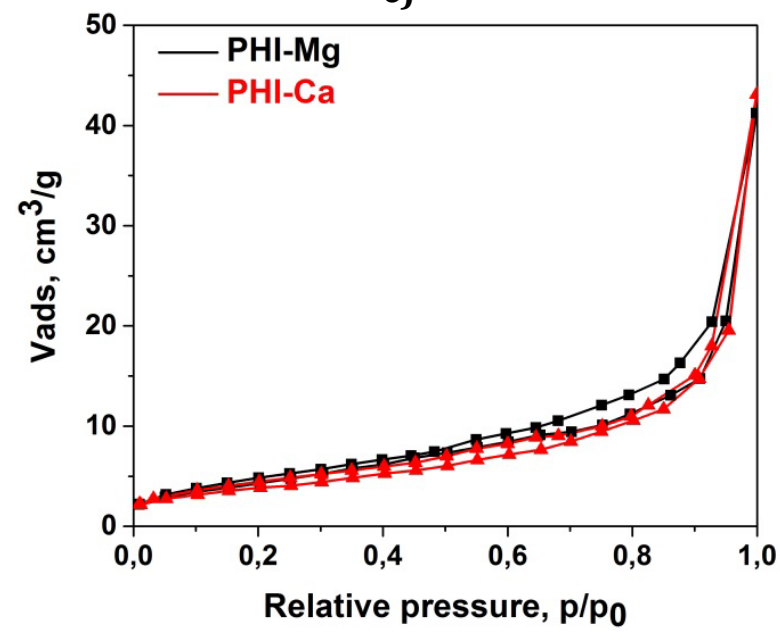

b)

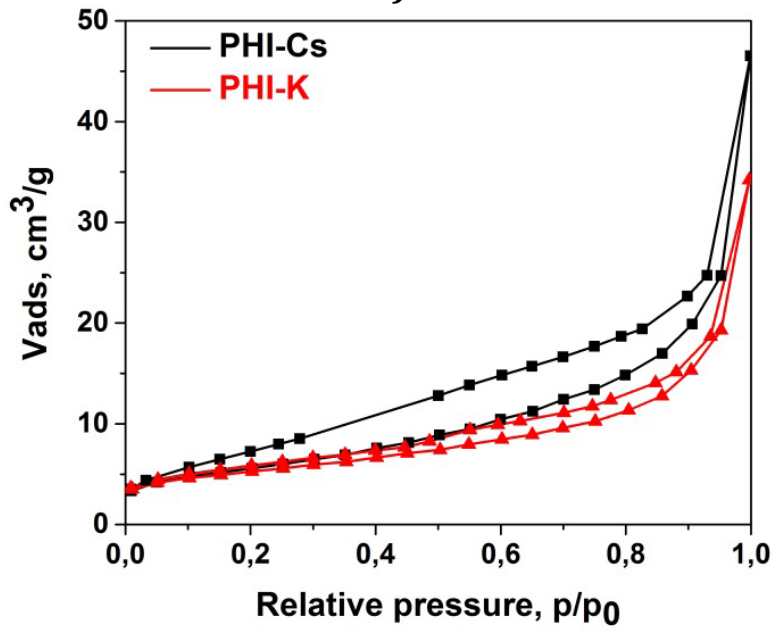

d)

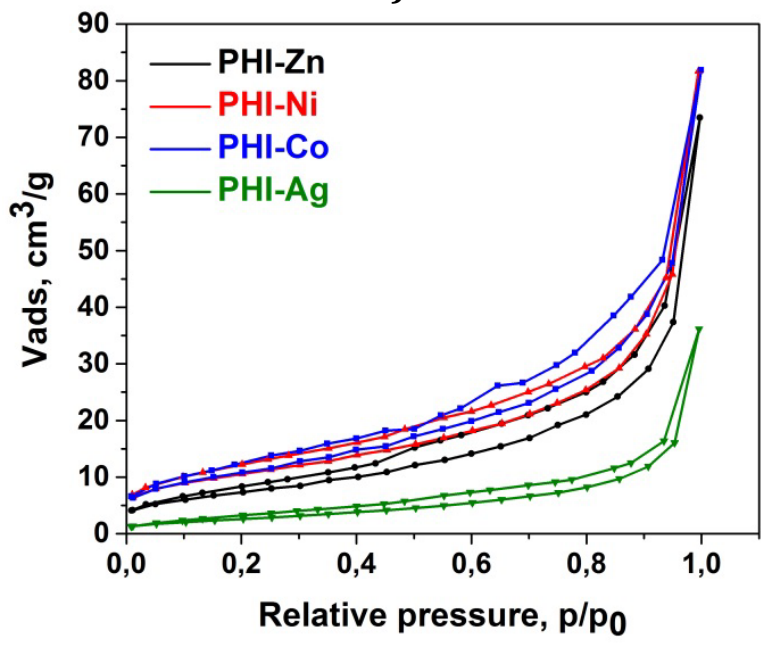


e)

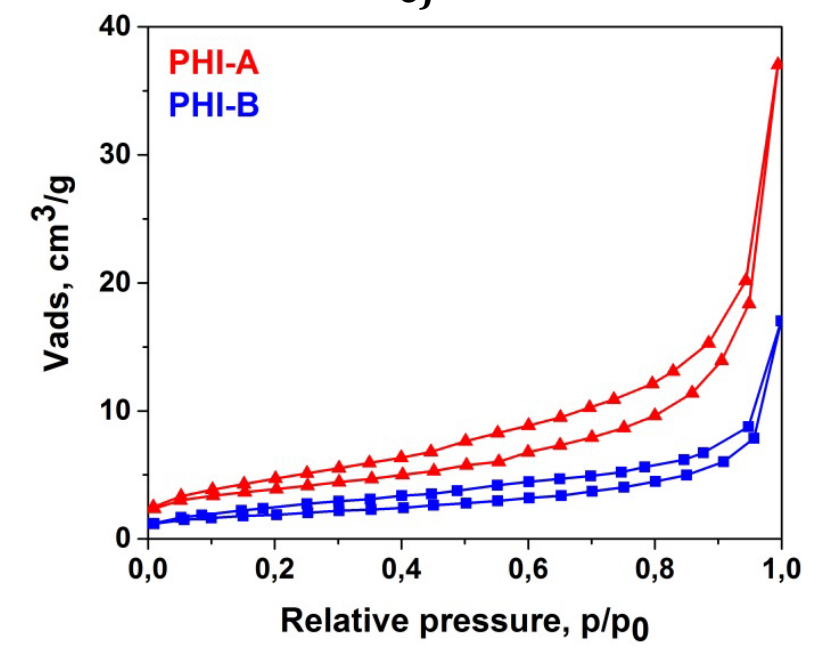

Figure S8. $\mathrm{N}_{2}$ sorption isotherms of the PHI-M materials.

a)

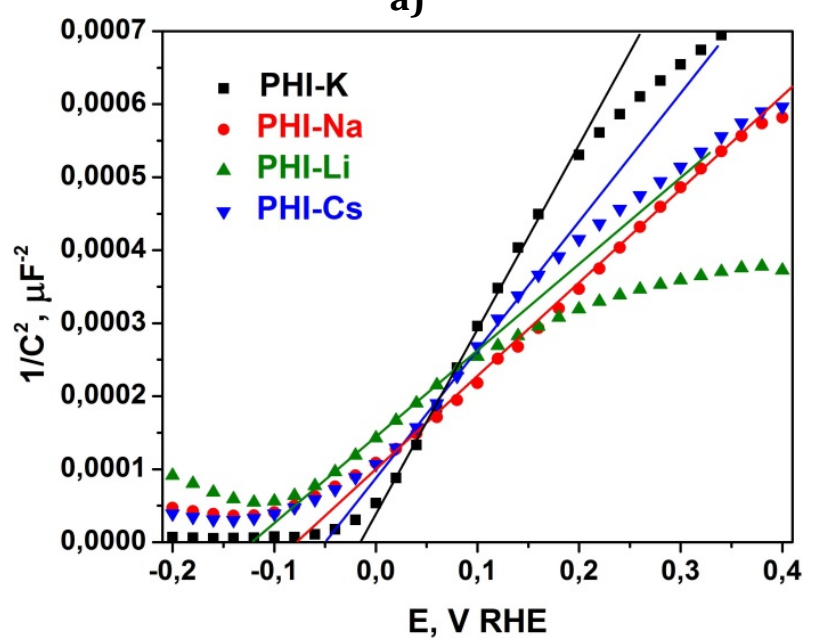

b)

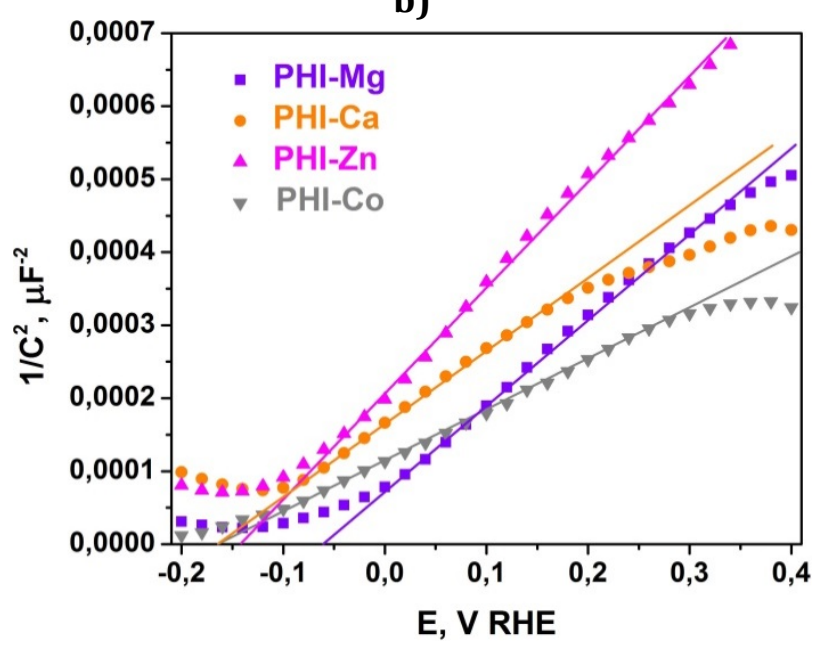

Figure S9. Mott-Schottky plots of PHI-M products. 


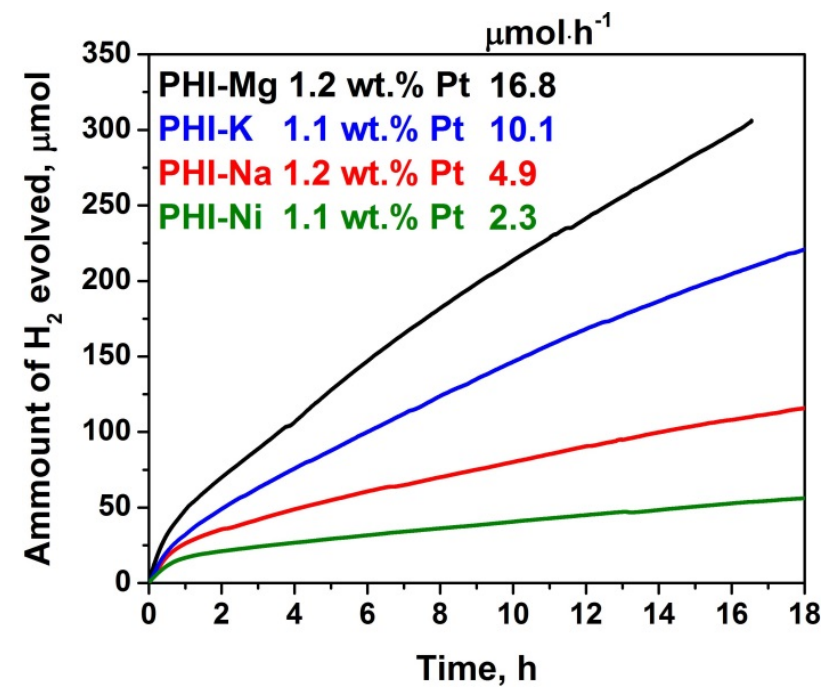

Figure S10. Time dependent hydrogen evolution over 1\%Pt@PHI-M products upon irradiation with visible light. Conditions: $50 \mathrm{~W}$ white LED array was used as a light source and TEOA as a sacrificial hole scavenger. 
All catalysts were collected after HER and analyzed for their stability by PXRD and FTIR spectroscopy (Figure S11) and elemental analysis (Table S4). Typically, a slight to significant decrease of the metal content was detected in the studied catalysts (Table S4). This is due to the concentration gradient of metal ions in the photocatalyst-solution system, which leads to partial replacement of metal ions in PHI-M with protons. The process is further assisted by TEOA that acts as a chelating agent and facilitates metal cation elimination from the solids. Indeed, a number of TEOA complexes with mono- [Polyhedron, 16, 12, 1997, 2087-2092; J. C. Voegele, J. Fischer, R. Weiss. Acta Cryst. 1974, B30, 62-65] and divalent metals [A. A. Naiini, J. Pinkas, W. Plass, V. G. Young Jr., J. G. Verkade., Inorg. Chem., 1994, 33 (10), 2137-2141] are known. The metal content drop is the most significant for the small-size alkali metals, i.e. lithium and sodium, so that only $39 \%$ and $43 \%$, respectively, of the original metal remains in the structure after 20 hours of HER test. The decrease of metal content is less pronounced for the large alkali metals, alkaline earth metals and $d$-metals. Ag and $\mathrm{Zn}$ are found to remain in full in the structure suggesting high thermodynamic stabilities of the corresponding solids. The FTIR spectra of PHI-M before and after HER tests are essentially similar (Figure S11). Only minor changes i.e. decrease of the absorption band intensity or slight shift of the band position, that are consistent with the partial replacement of metal ions with protons and improvement of the structural order, are observed. The spectrum of PHI-Co after HER indicates the disappearance of $\mathrm{NH}_{2}$-groups observed in the solid before the test pointing to the side red-ox reactions mentioned above.

a)

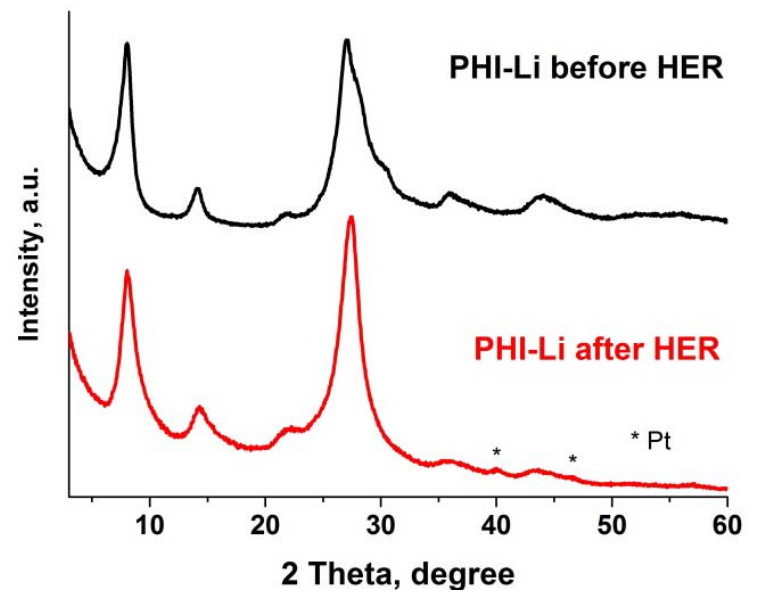

c)

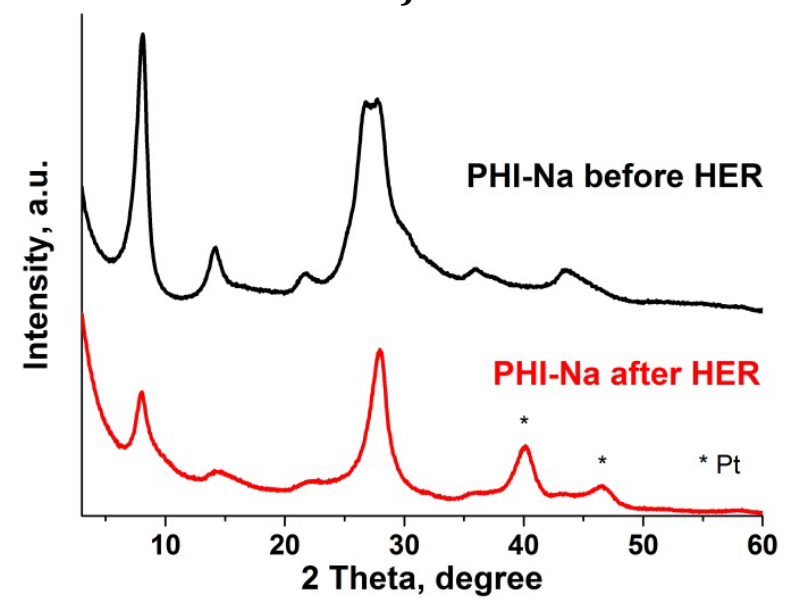

b)

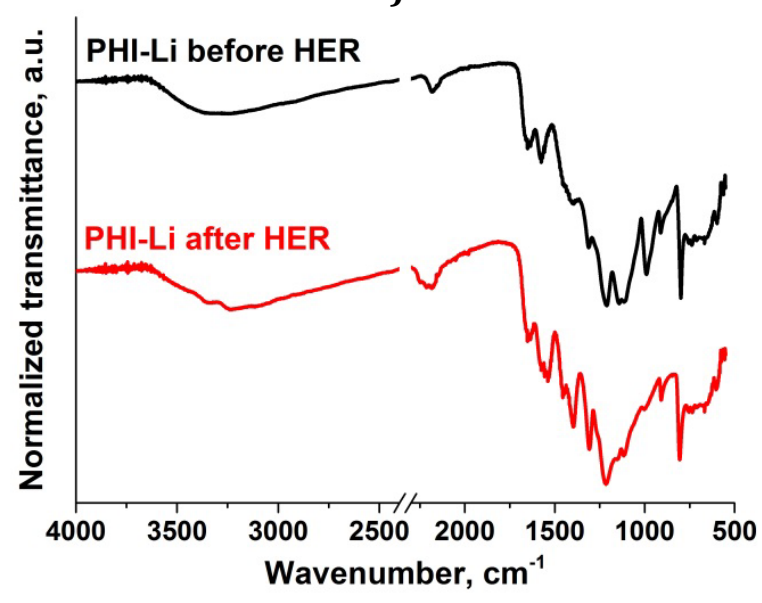

d)

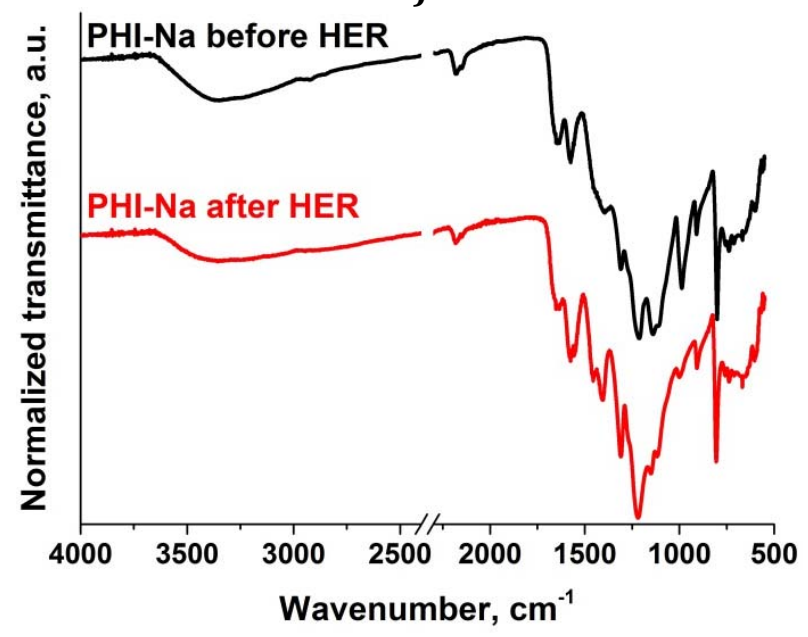


e)

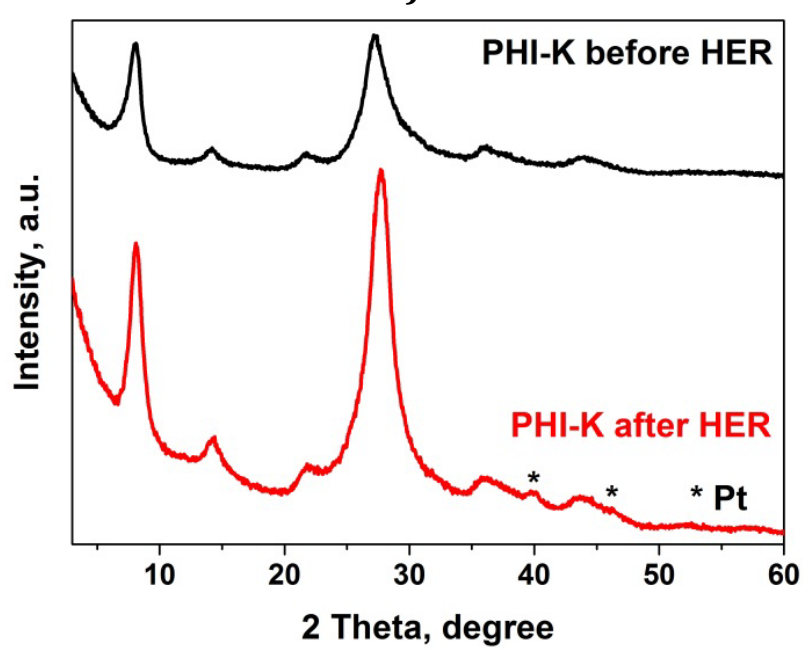

g)

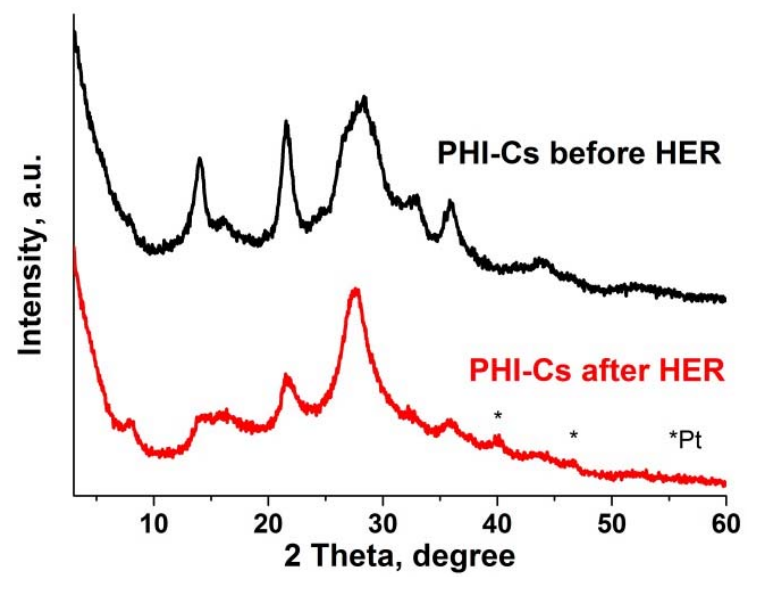

i)

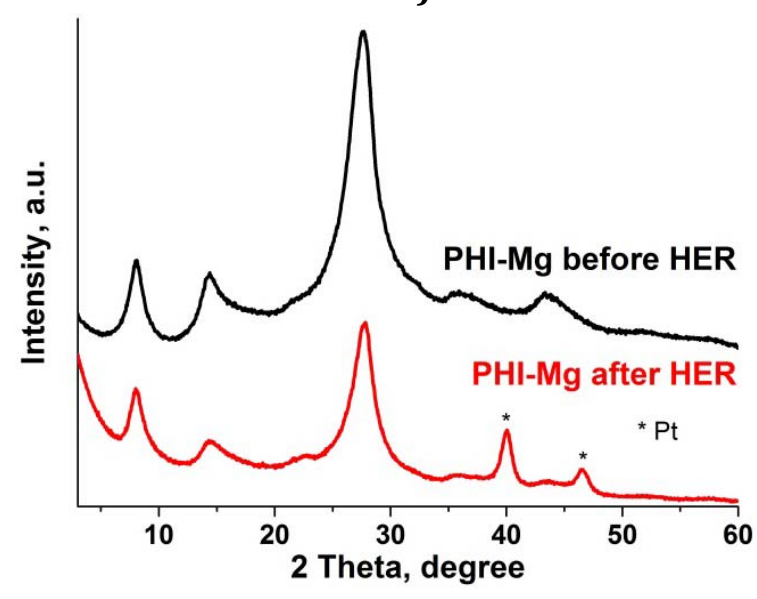

f)

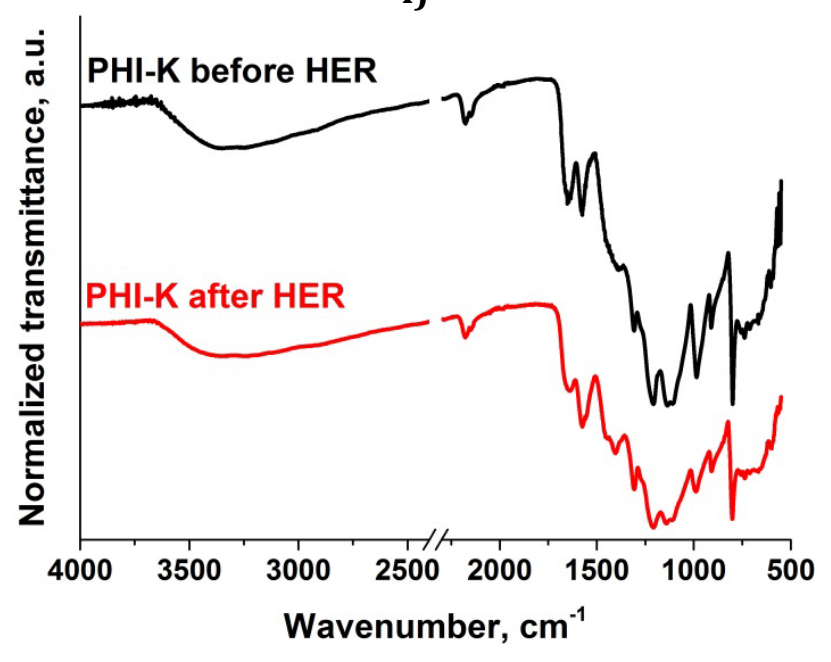

h)
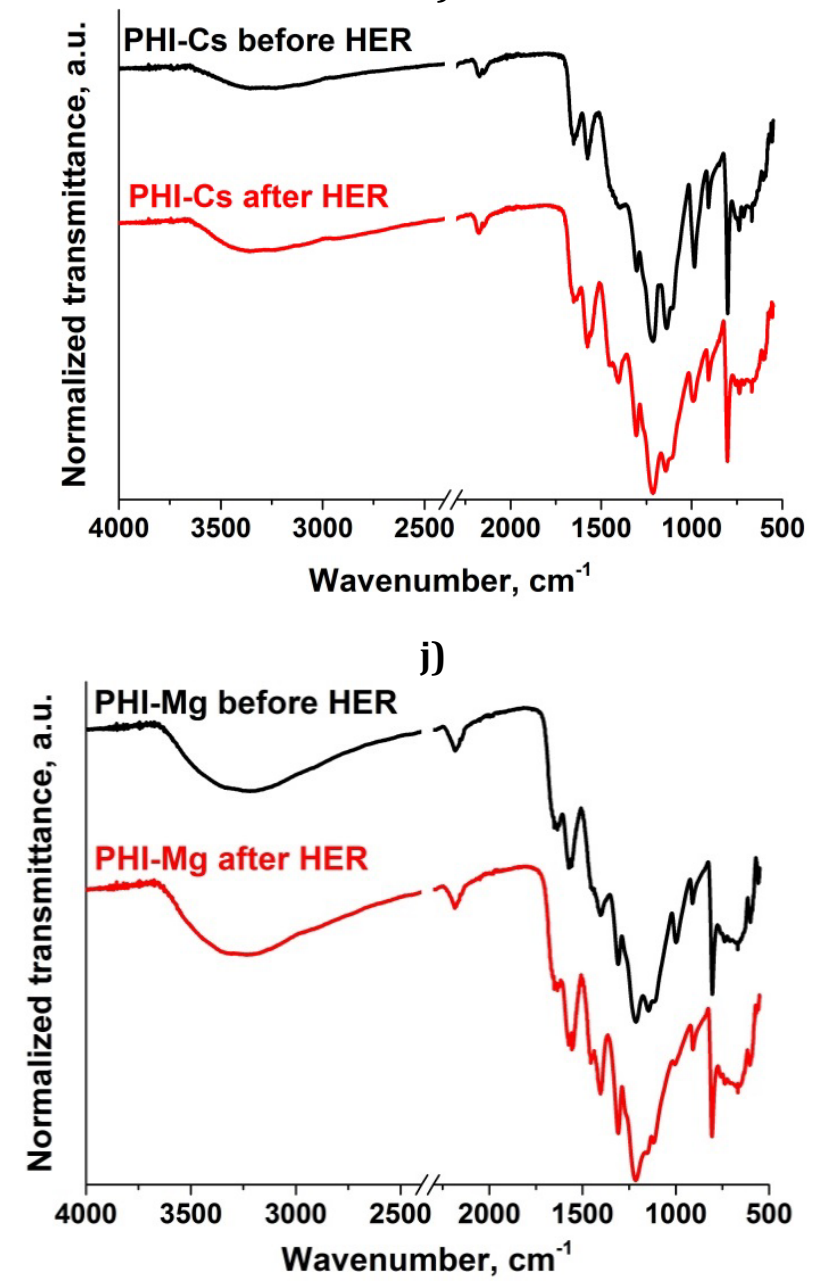
k)

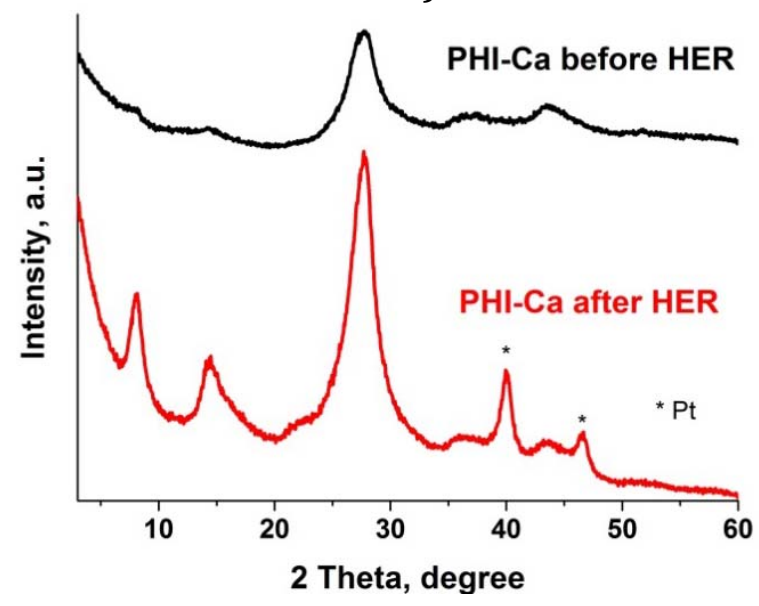

m)

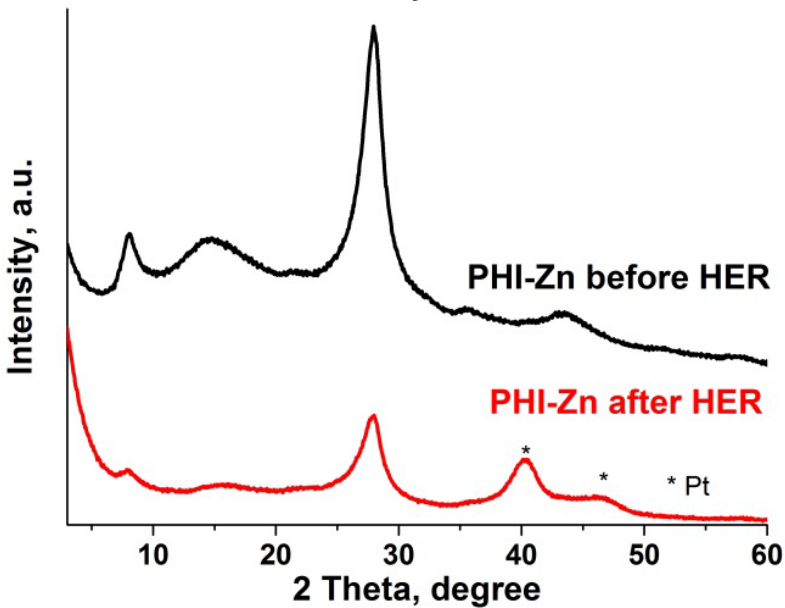

o)

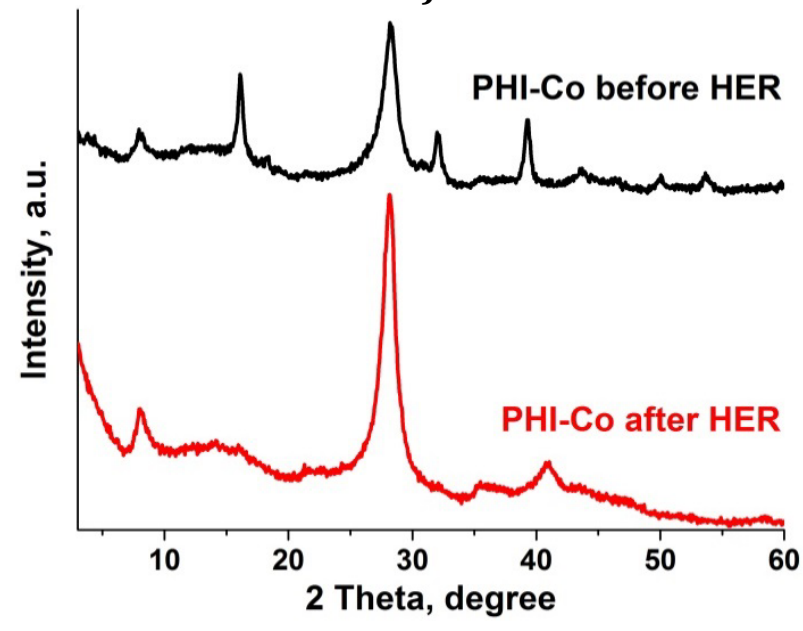

l)

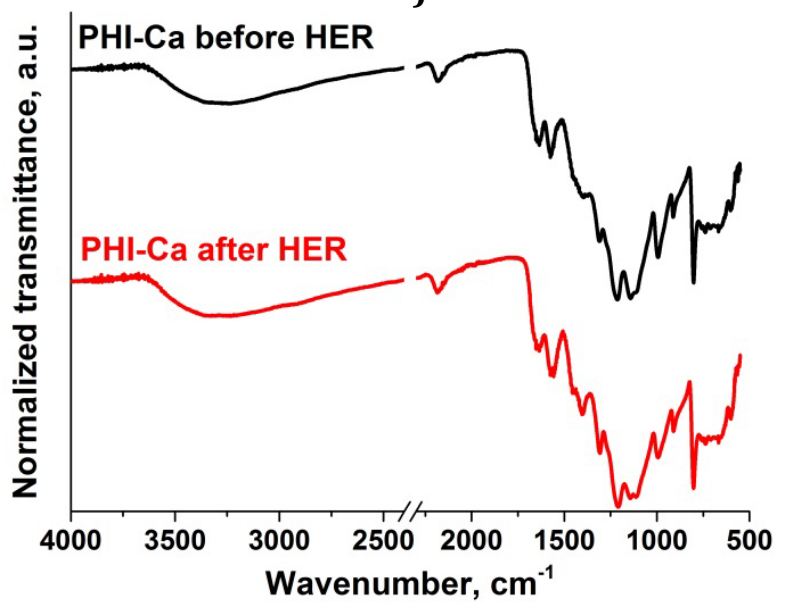

n)

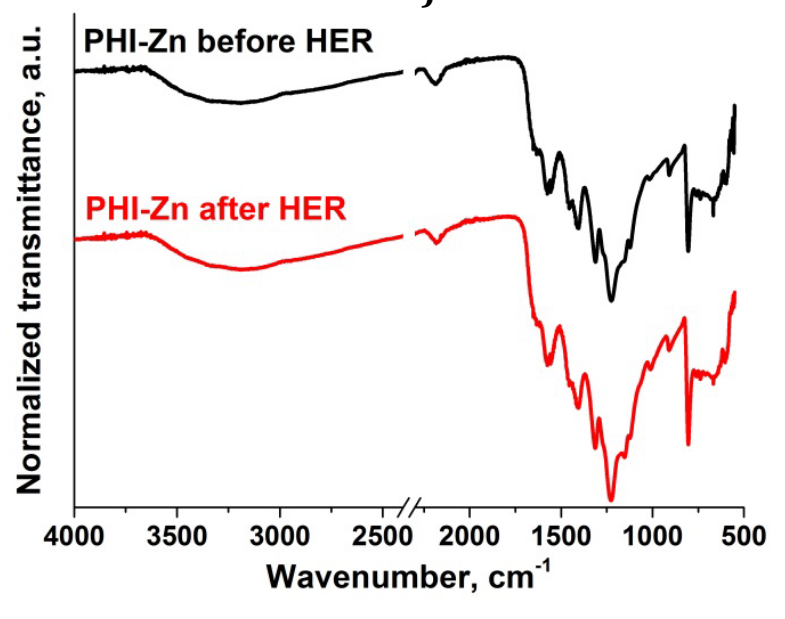

p)

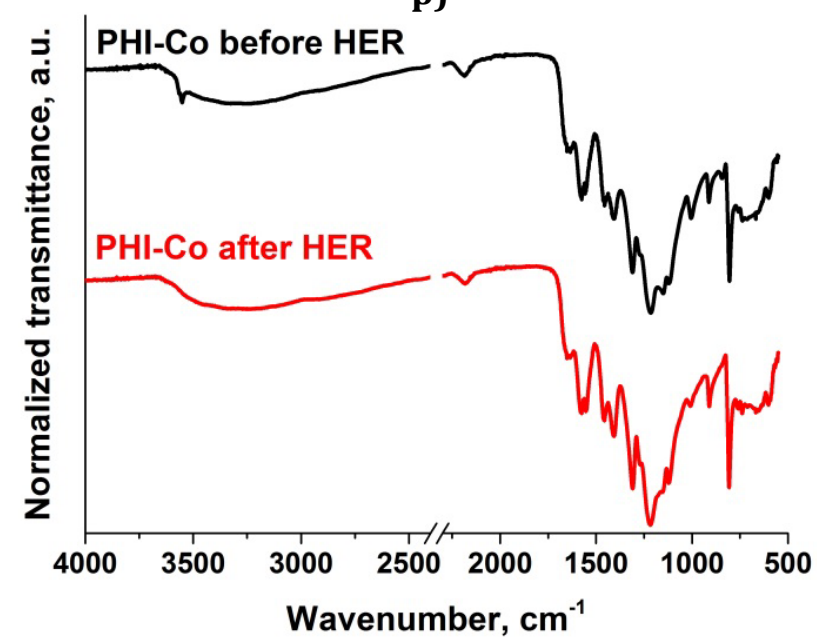


q)

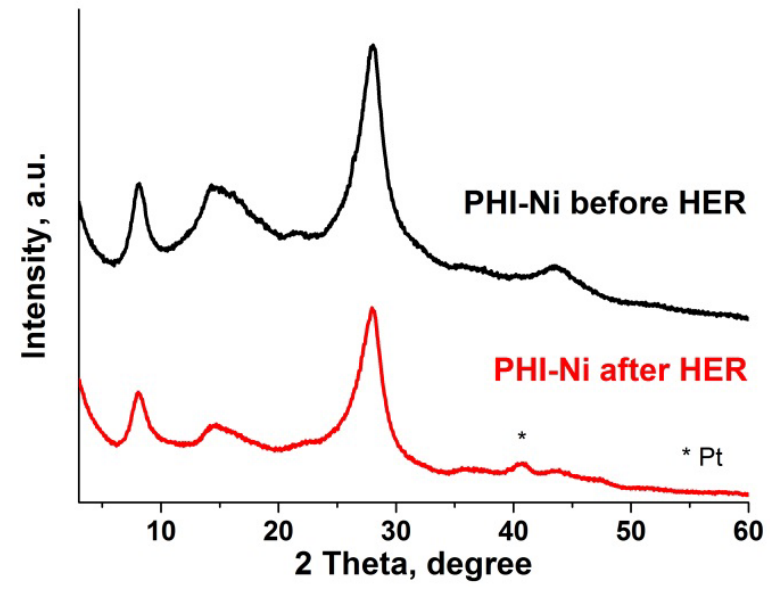

s)

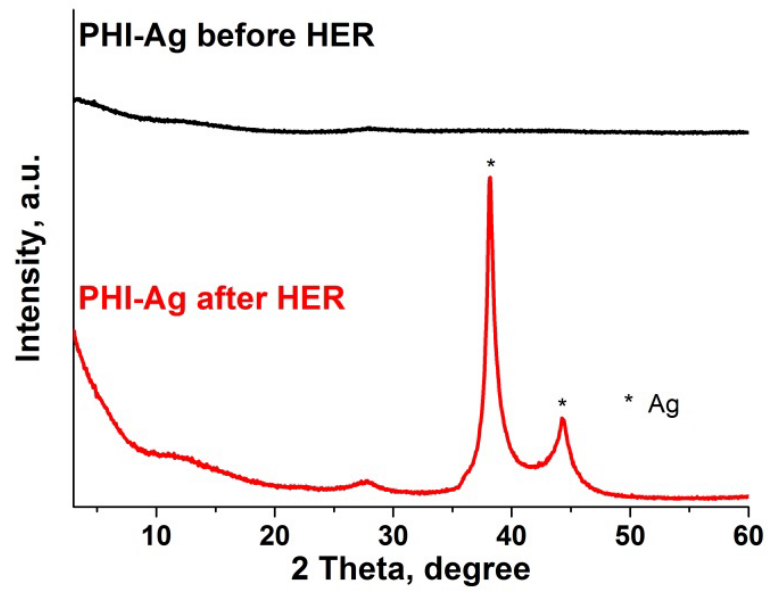

r)

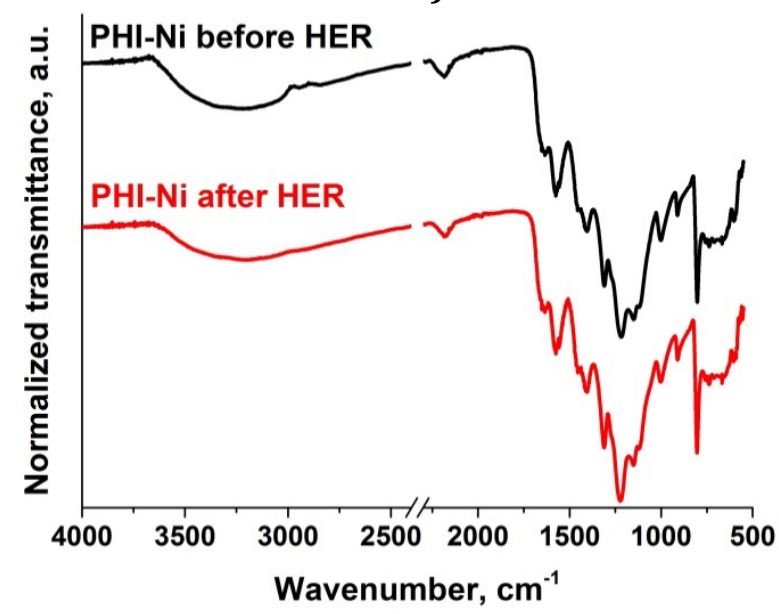

t)

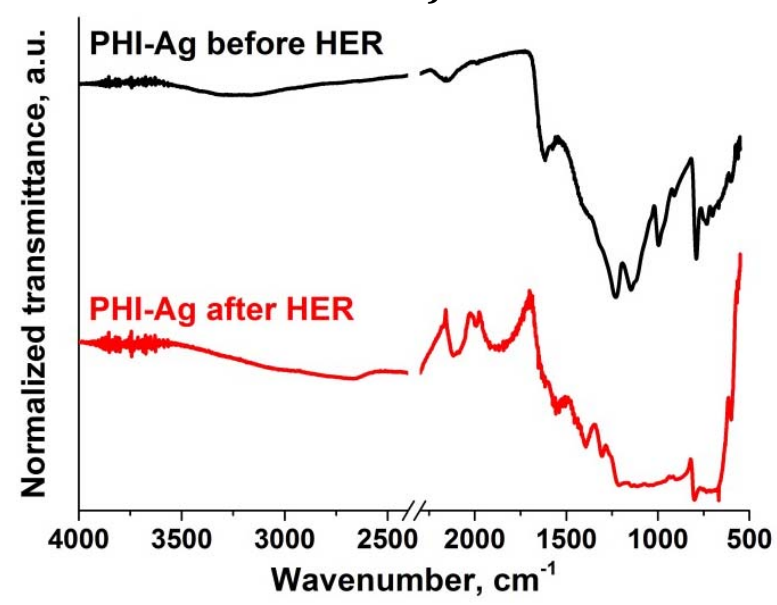

Figure S11. Comparison of the PXRD patterns and FTIR spectra of the PHI-M photocatalysts before and after HER: PHI-Li (a,b), PHI-Na (c,d), PHI-K (e,f), PHI-Cs (g,h), PHI-Mg (i,j), PHI-Ca (k,l), PHI-Zn (m,n), PHI-Co (o,p), PHI-Ni (q,r), PHI-Ag (s,t). 
The sustainability of hydrogen production was assessed in a long-duration photocatalytic test using PHI-Mg as a photocatalyst (Figure S12a). A decrease of activity from 25.1 to $17.1 \mu \mathrm{mol} \mathrm{H}_{2} /$ hour is observed after 60 hours of irradiation. It can be explained by the contamination of the catalyst surface with the products of TEOA oxidation, and by the partial removal of $\mathrm{Mg}^{2+}$ ions from the structure (vide infra). In order to distinguish between those two factors, a sample recovered after longduration test PHI-Mg was thoroughly washed with water and again used in HER, without the addition of fresh Pt NP precursor. The catalyst recovered $87 \%$ of its original activity producing $21.8 \mu \mathrm{mol}$ $\mathrm{H}_{2}$ /hour (Figure S12b). In principle, the loss of metal ions during HER can be prevented by adding small quantities of the corresponding metal salt in the reaction mixture.

a)

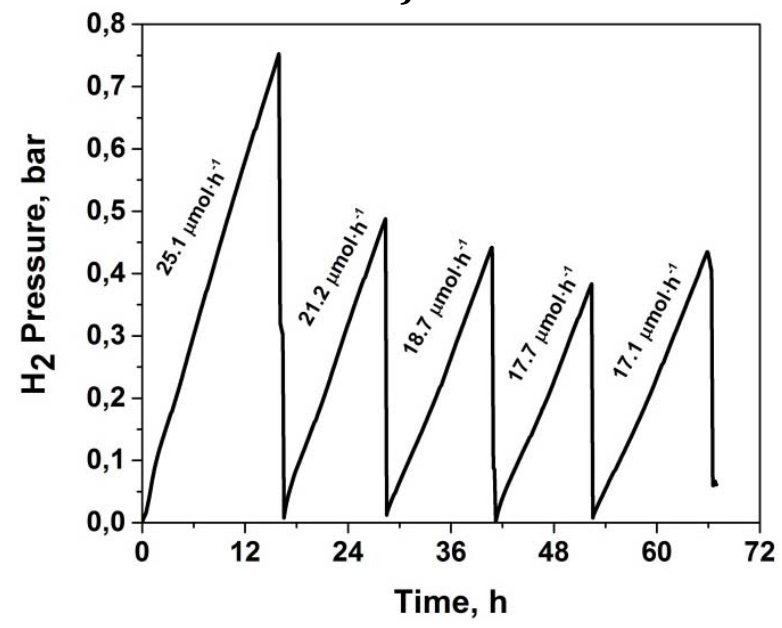

b)

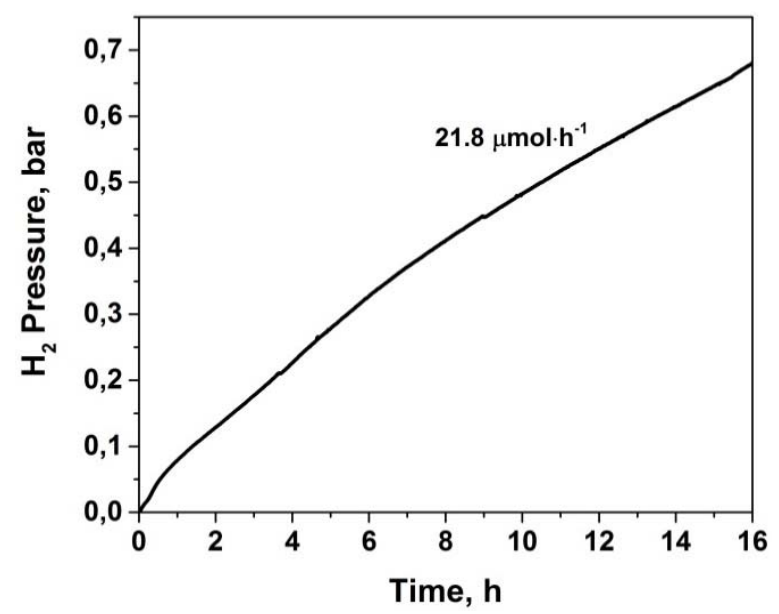

Figure S12. Stability tests performed for PHI-Mg catalysts: long-duration test (a) and recovered catalysts test (b).

The oxidation stability of PHI-M photocatalysts was assessed by analysing the headspace of the reaction mixtures after 18-20 hours of HER tests using GC-MS. In all the cases, hydrogen was the only gaseous product of the reaction, and no $\mathrm{CO}_{2}$ which would indicate the decomposition of TEOA or photocatalysts, was detected (Figure S13):

a) before reaction

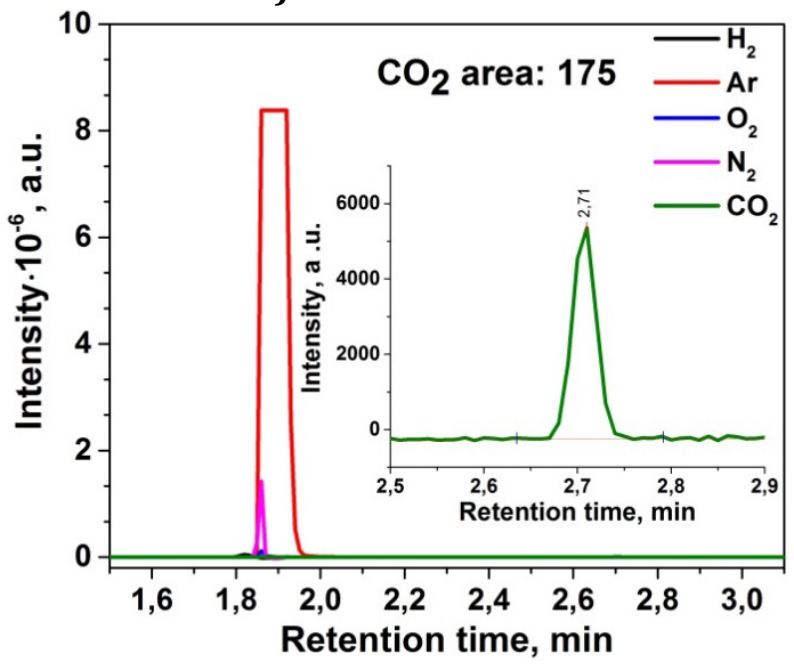

b) PHI-Li

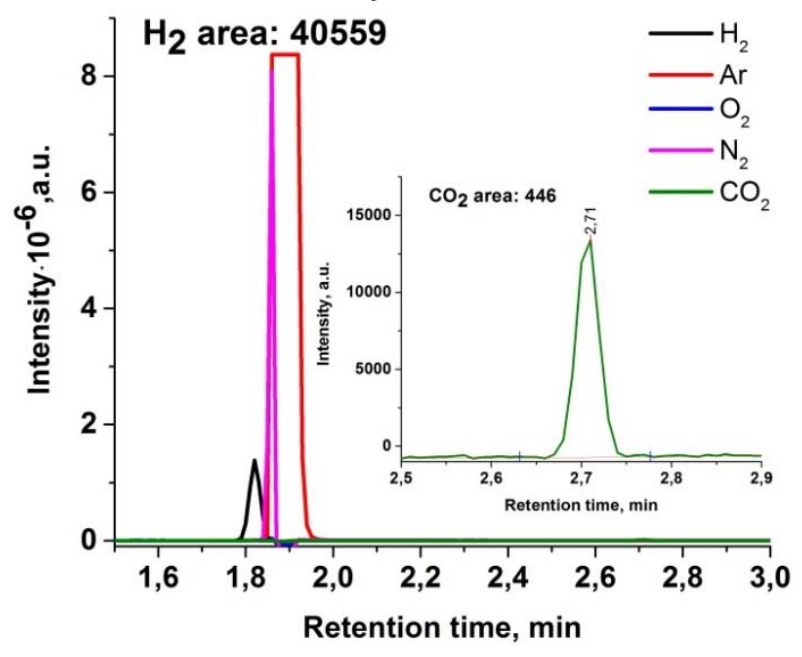


c) PHI-Cs

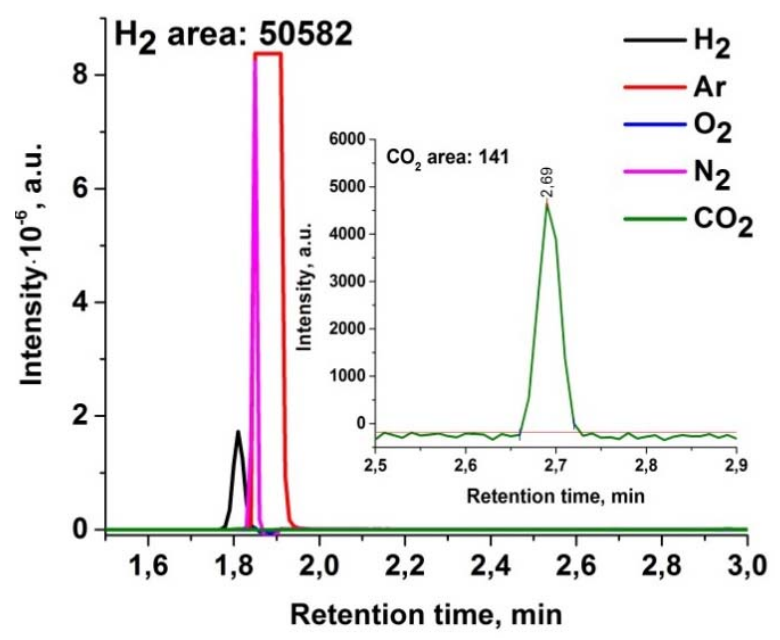

e) PHI-Ca

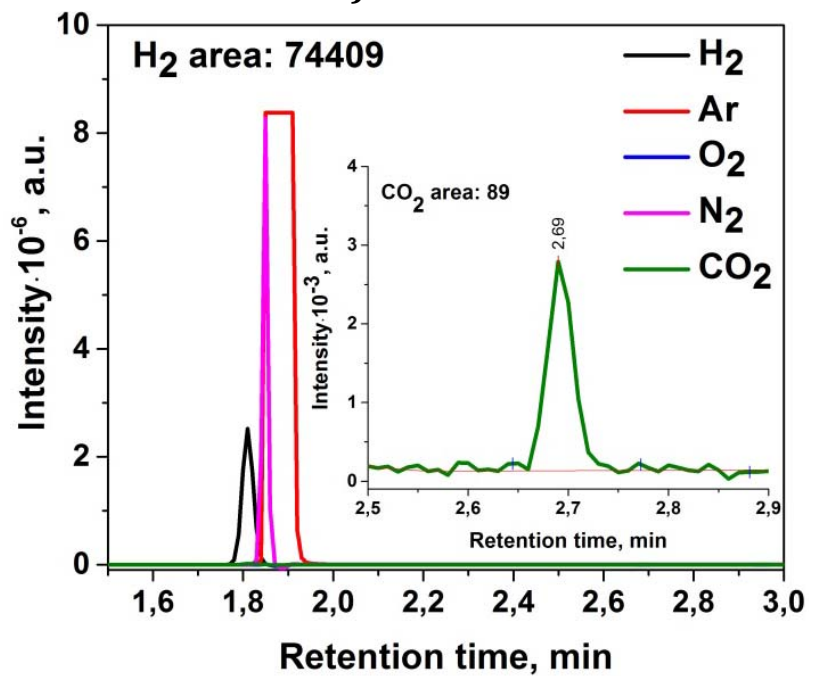

d) PHI-Mg

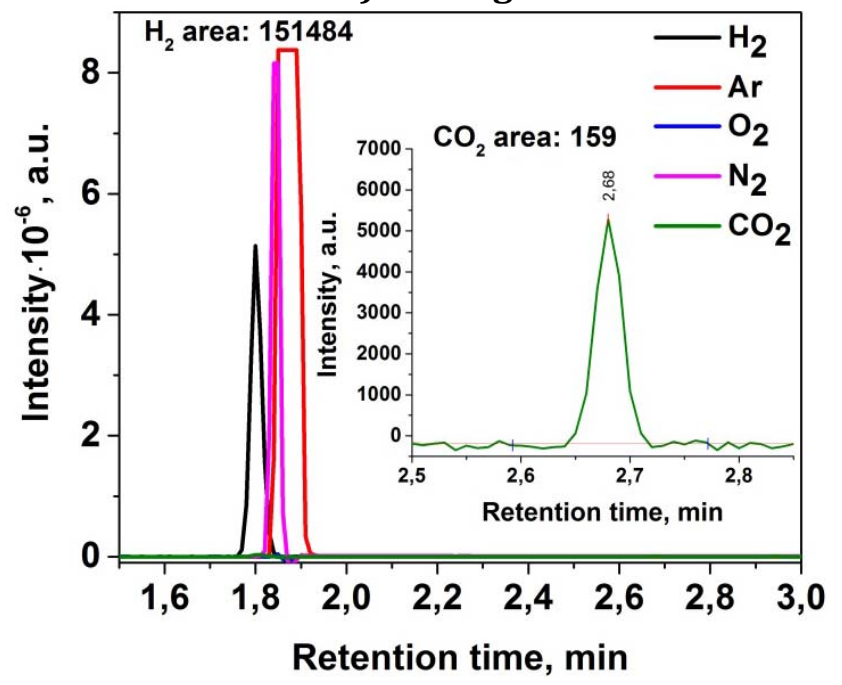

f) PHI-Zn

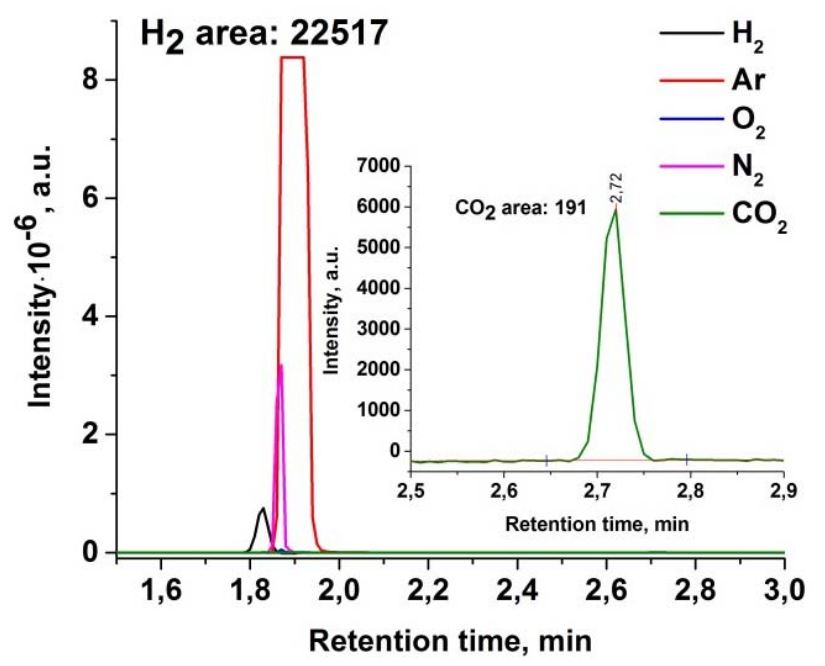

g) PHI-Co

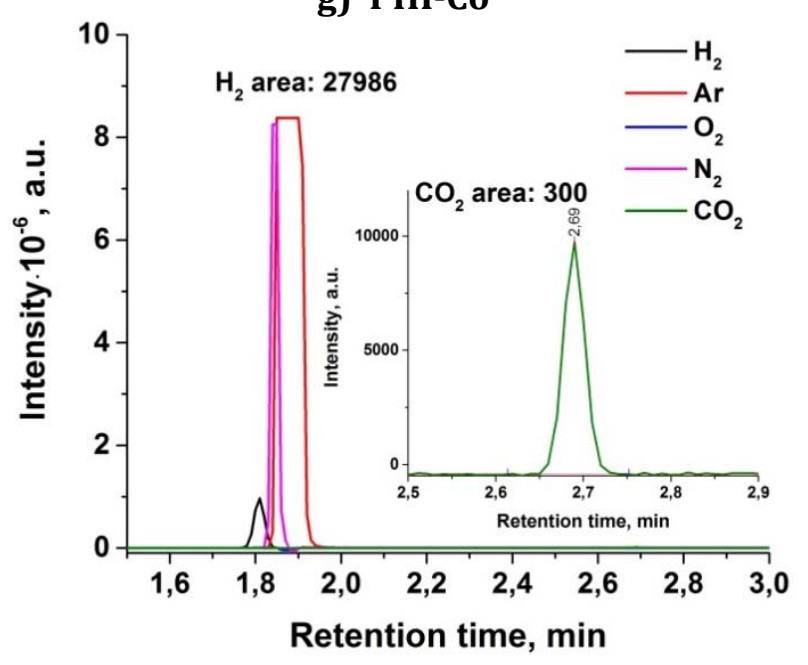

Figure S13. Composition of the reactor head space gas mixtures as analysed by GC-MS before irradiation (a), and after HER tests using PHI-Li (b), PHI-Mg (c), PHI-Ca (d), PHI-Cs (e), PHI-Zn (f), and PHI-Co (g) as photocatalysts. Trace amounts of $\mathrm{CO}_{2}$ originate from the small leaks during headspace sample handling and from the GC-MS measurement set-up. 
Table S4. EDS analysis of the PHI-M catalysts after HER.

\begin{tabular}{|c|c|c|c|c|c|c|c|}
\hline \multirow[t]{2}{*}{ Catalyst } & \multicolumn{6}{|c|}{ Element content, wt. \% } & \multirow{2}{*}{$\begin{array}{c}\mathbf{M a}^{\mathrm{a}}, \mathbf{w t} . \\
\%\end{array}$} \\
\hline & C & $\mathbf{N}$ & $\mathbf{M}$ & $\mathbf{0}$ & $\mathrm{Cl}$ & Pt & \\
\hline PHI-Li & 35.20 & 57.03 & 0.86 & 4.65 & 1.72 & 0.54 & $2.22^{\mathrm{b}}$ \\
\hline PHI-Na & 36.36 & 54.14 & 2.83 & 3.56 & 0.10 & 3.01 & 6.60 \\
\hline PHI-K & 34.01 & 54.14 & 7.34 & 3.95 & 0.01 & 0.94 & 11.88 \\
\hline PHI-Cs & 29.84 & 42.96 & 24.28 & 1.41 & 0.27 & 1.23 & 32.53 \\
\hline PHI-Mg & 33.64 & 51.76 & 2.19 & 7.87 & 2.98 & 1.57 & 2.70 \\
\hline PHI-Ca & 32.11 & 51.80 & 6.27 & 6.30 & 2.18 & 1.34 & 6.72 \\
\hline PHI-Zn & 29.10 & 41.75 & 18.62 & 7.39 & 1.22 & 1.91 & 19.07 \\
\hline PHI-Co & 34.99 & 53.30 & 4.38 & 4.60 & 1.02 & 1.71 & 9.51 \\
\hline $\mathrm{PHI}-\mathrm{Ni}$ & 35.67 & 53.01 & 5.09 & 5.35 & 0.22 & 0.66 & 6.06 \\
\hline PHI-Ag & 19.08 & 32.57 & 39.02 & 4.17 & 0.00 & 5.16 & 38.89 \\
\hline
\end{tabular}

a metal content in the corresponding catalyst before HER; b ICP data. 
The photocatalytic activity of PHI-D in water reduction reaction was found to be $17.3 \mu \mathrm{mol}$ $\mathrm{H}_{2}$ /hour at 0.79 wt.\% Pt loading, illustrating the efficiency of the dispersion procedure (Figure S14).

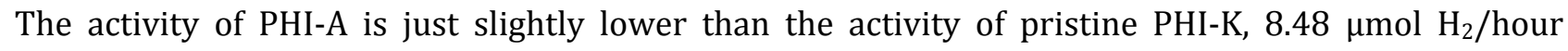
compared to $9.7 \mu \mathrm{mol} \mathrm{H}_{2}$ /hour that can be explained by slightly lower amount of photodeposited $\mathrm{Pt}$ ( 0.61 vs. 0.94 wt.\%). This finding clearly indicates that the presence of $\mathrm{K}^{+}$ions is not essential for catalytic activity in photocatalytic water reduction, since PHI-A contains no $\mathrm{K}^{+}$ions. PHI-B is more active in HER than PHI-K producing $13.7 \mu \mathrm{mol} \mathrm{H}_{2}$ /hour which is probably due to the increased $\mathrm{Pt}$ loading of $1.47 \mathrm{wt} . \%$. On the other hand, the solid recovered after PHI dissolution in sulphuric acid, PHI-SA is inactive in HER..

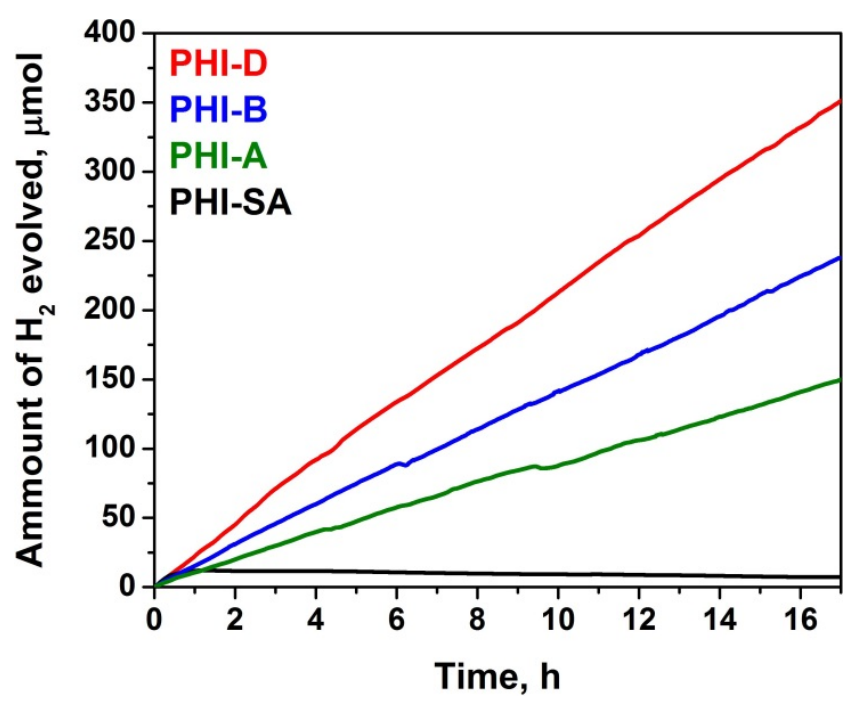

Figure S14. Hydrogen evolution over PHI-A, PHI-B, PHI-SA and PHI-D photocatalysts upon visible light irradiation (50 W white LED) using triethanolamine as a sacrificial agent and photodeposited Pt as a co-catalyst. 